\title{
Leaf-mining moths (Lepidoptera) of the Biedrusko military area in western Poland
}

\author{
Urszula WALCZAK \\ Department of Systematic Zoology, Faculty of Biology, Adam Mickiewicz University, \\ Umultowska 89,61-614 Poznań, Poland; e-mail: urszulaw@amu.edu.pl,
}

\begin{abstract}
Results of the research on the leaf-mining moths (Lepidoptera) carried out in the Biedrusko military area in 1997-2008 are presented. The investigation showed great species richness of the moths. 258 species have been recorded in the military area, which makes about $47 \%$ of this fauna reported from Poland. 24 species are new to Greater Poland Voivodeship and several were reported from few, scattered localities. The most interesting species are described in details. New data on the biology of Syncopacma ochrofasciella are also given.
\end{abstract}

Key words: Lepidoptera, leaf-miners, W Poland, military area, diversity, faunistics, new records

\section{INTRODUCTION}

The military grounds in Biedrusko cover the area of about 7200 ha to the north of Poznan. The area has been used as military grounds for over a hundred years and thus it shows little anthropogenic transformation. The "Biedrusko" Protected Landscape Area has been established in 1995 to protect this naturally valuable area. Later this area has been included into the European Ecological Natura 2000 Network as a Special Area of Conservation (site code PLH300001).

The earliest information on the nature of the Biedrusko military ground comes from the beginning of the 20th century (Dorn 1919). Subsequent papers were published only in the beginning of $1990 \mathrm{~s}$. so after the political transformations in Poland. when it was possible to perform studies over this area. However, despite the attractiveness of the area. closeness to the academic centres and approval of the military authorities. the grounds have not been comprehensively studied yet. The hitherto studies concerned the flora and selected plant associations (Borysiak \& Brzeg 1994; Wyrwol 1994; Zielińska 1994; Rakowski 1996; Stachnowicz 1996. 1997; Borysiak et al. 1998). vertebrates. mostly the birds. (Winiecki 1992) and insects: butterflies (Walczak 2002), beetles (e.g. Konwerski 2002; Konwerski \& Sienkiewicz 2005). aphids (Wilkaniec et al. 2008) and parasitic wasps (Piekarska-Boniecka \& Wilkaniec 2008). The mining moths of this area have not been studied yet.

The main aim of the study was to recognise the species composition of mining moths of this area and their environment selectivity in relation to vegetation type.

\section{STUDY AREA}

The area has diversified post-glacial landscape. The central part is the moraine plateau bordering on the south with the hills of the terminal moraine of Poznan Phase of the Baltic Glaciation. On the north and east the area covers fragments of the Warta River valley (Bartkowski 1962). To a great extent the area is covered with forest. However. parts of these forested grounds do not present high natural value mainly because of incorrect forest management that has led to degradation of habitats and degeneration of vegetation. A large part 
of the area is grown with pine monocultures with some deciduous species; the ground is often occupied by reed grass Calamagrostis epigeios (L.) Roth and various species of the genus Rubus L. The contribution of oak forests is relatively large. and is represented mainly by acidophilous oak forests Calamagrostio-Quercetum petraeae (Borysiak \& Brzeg 1994). There are well-developed ash-alder forests Fraxino-Alnetum. which greatest cluster is localised near the Glinnowiecki Brook and in the valley of the Pólnocny Ditch. In the northern part of the military grounds. along the old river beds, there are patches of elm-ash forest Ficario-Ulmetum campestris (Wyrwol 1994). About 5\% of the forested area is covered by alder forests. including fertile alder forest Ribo nigri-Alnetum and oligotrophic woodland Sphagno squarrosi-Alnetum (Borysiak \& Brzeg 1994).

Thicket communities are widespread over the area; the greatest part is occupied by the thickets with common broom Cytisus scoparius (L.) Link, responsible for the specific look of the central part of the area (Borysiak \& Brzeg 1994), deforested for the purpose of military training. Thickets with blackthorn and hawthorn Pruno-Crataegetum, occurring as shrubs in forest edge or more often individually in the open landscape. plays also an important role. Sallow thickets Salicetum pentandro-cinereae are common along the watercourses and on the banks of lakes and small ponds. Less frequent are the filbert thickets Euonymo-Coryletum and thickets with spindle-tree, cranberry-tree and red dogwood Euonymo-Cornetum (Borysiak \& Brzeg 1994). Typical elements of the weakly transformed landscape are the ecotone communities: herbaceous vegetation of forest edges and thicket fringes. Among the nitrophilous forest edge communities the most important are the hygrophilous communities from the allion Convolvulion sepium, related to the Warta River valley and local depressions. Xerothermophilous forest edge communities representing the class Trifolio-Geranietea sanguinei are more frequently met and better developed. They grow along the forest edges and accompany the thickets of the class Rhamno-Prunetea. in particular thickets with blackthorn and hawthorn Pruno-Crataegetum (Borysiak \& Brzeg 1994).

Vast area of the military grounds is covered with meadows of the class MolinioArrhenatheretea, left after former use. Semi-moist purple moor-grass meadows Molinietum medioeuropaeum. represented by a very rare thermophilous and calcareous form, are of particular value (Borysiak \& Brzeg 1994). These meadows are found mainly in valleys along the watercourses. A relatively large group of Molinia meadows is localised on the Pólnocny Ditch and its tributaries, between Chludowo and Cielak Forest and on the southern margin of the Sarnia Meadow (Stachnowicz 1996).

The wide open areas of the central part of the military grounds are covered with grassland communities. The site is dominated by large extents of psammophilous grassland DianthoArmerietum. although its patches are often poorly developed and of low value (Borysiak \& Brzeg 1994). Smaller areas are occupied by xerothermic grasslands representing three associations identified. Particularly interesting are the grasslands of the meadow steppe type. classified by Rakowski (1996) to the community Carlina vulgaris-Ononis spinosa. These flowery grasslands are floristically the richest over the whole military grounds. The synanthropic, segetal and ruderal communities occupy relatively small area and have not been subjected to detailed phytosociological studies. The military grounds border on the arable fields only along the western edge and the weed communities are rather poorly developed there (Borysiak \& Brzeg 1994).

Leaf-mining moths were studied at 47 sites (Fig. 1):

1. Radojewo. Piolunowa street - roadside trees and ruderal vegetation

2. pine greenwood

3. left-bank terrace of Glinnowiecki Brook. ash-alder forest Fraxino-Alnetum 

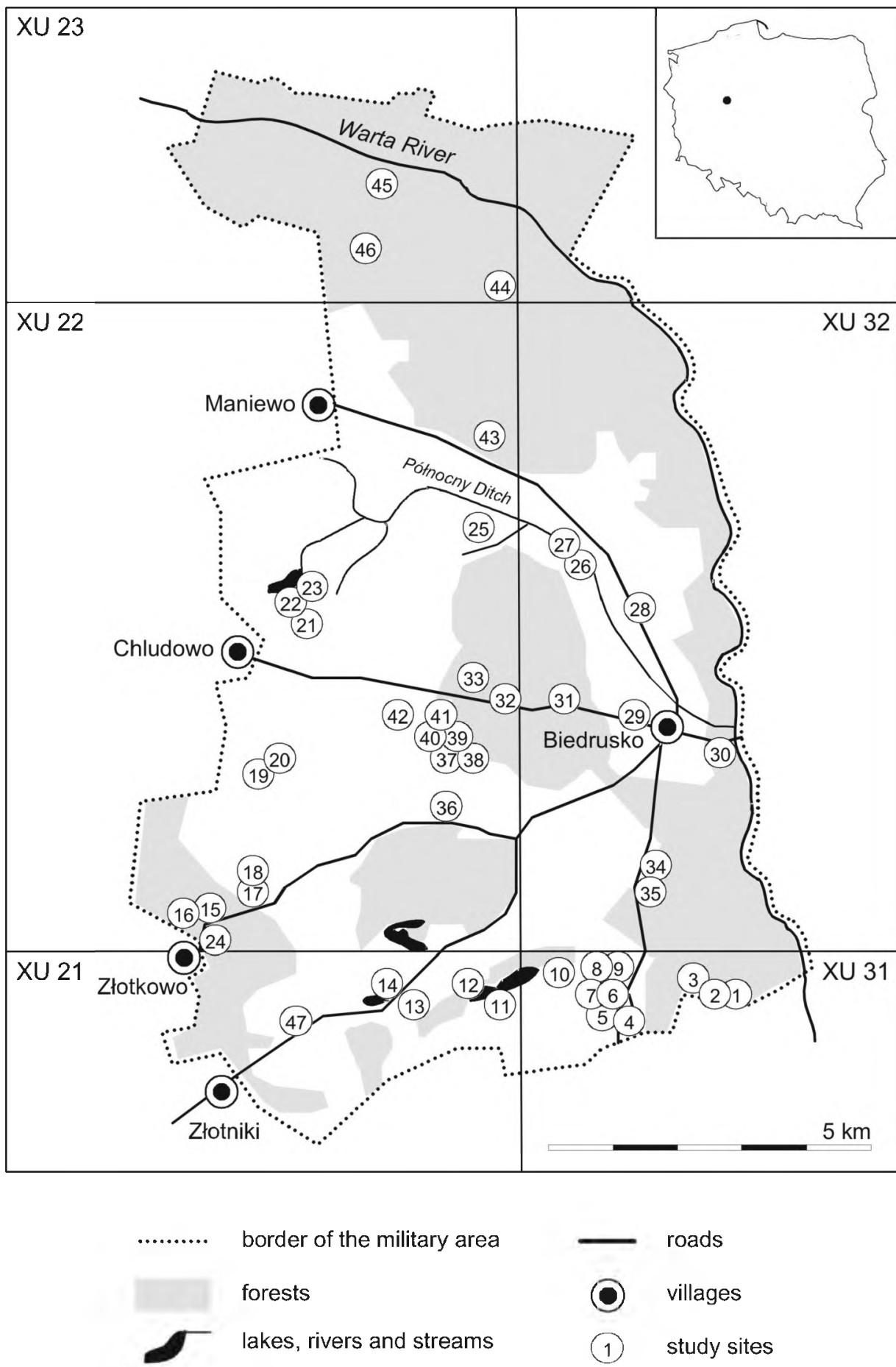

Fig. 1. Location of the study sites in the UTM grid system. 
4. psammophilous grassland of the class Koelerio-Corynephoretea between road fork Radojewo - Biedrusko

5. trees and herbaceous vegetation of the class Artemisietea along the road BiedruskoRadojewo

6. xerothermic grassland of the class Festuco-Brometea

7. psammophilous grasslands, herbaceous vegetation, willow, birch and alder thickets in the vicinity of tank road

8. psammophilous grassland of the class Koelerio-Corynephoretea in the vicinity of tank road

9. Biedrusko-Radojewo road - ruderal and forest edge vegetation of the class Artemisietea, thickets and roadside trees

10. psammophilous grassland of the class Koelerio-Corynephoretea, forest edge vegetation of the class Artemisietea, thickets, roadside trees and fertile alder forest Ribo nigri-Alnetum

11. southern shore of Glinnowieckie Lake - fertile alder forest Ribo nigri-Alnetum

12. north-western shore of Glinnowieckie Lake - oak-hornbeam forest Galio silvaticiCarpinetum

13. Bogusławski Road - ruderal and forest edge vegetation of the class Artemisietea, thickets and fertile alder forest Ribo nigri-Alnetum

14. northern shore of no-name little lake - xerothermic vegetation of the class FestucoBrometea

15. oligotrophic woodland Sphagno squarrosi-Alnetum, a patch adjoining the northern part of transitional peat-bog of the Gogulec Reserve

16. sallow thickets Salicetum pentandro-cinereae surrounding a transitional peat-bog of the Gogulec Reserve and rushes of the class Phragmitetea

17. Zlotkowo-Biedrusko road - psammophilous grassland of the class KoelerioCorynephoretea in an old gravel-pit

18. heath communities of the order Calluno-Ulicetalia and birch thickets

19. S slope of Napoleon Hill - xerothermic grassland of the class Festuco-Brometea on the south-facing slope

20. xerothermic grassland of the class Festuco-Brometea, meadows of the class MolinioArrhenatheretea, thickets of the class Rhamno-Prunetea

21. sallow thickets Salicetum pentandro-cinereae between Chludowo and Cielak Forest

22. ground road leading to Chludowskie Lake - common broom thickets

23. S-E shore of Chludowskie Lake - a mixture of grasslands, meadows and thickets

24. Zlotkowo - forest road and old, neglected orchard

25. grasslands of the class Festuco-Brometea, meadows of the class Molinio-Arrhenatheretea, thickets of the class Rhamno-Prunetea and sallow thickets Salicetum pentandro-cinereae along Pólnocny Ditch, to the north of the Lody Forest

26. Maniewska Road, ponds along Pólnocny Ditch - aquatic vegetation and rushes of classes Lemnetea, Potamogetonetea and Phragmitetea

27. meadows of the class Molinio-Arrhenatheretea, grasslands of the class KoelerioCorynephoretea, herb vegetation of the class Artemisietea and willow thickets

28. Maniewska Road, along Olszynka to the sentry box with barrier - roadside trees, thickets and herb communities of the class Artemisietea

29. Biedrusko - roadside trees, thickets and ruderal vegetation

30. left bank of Warta River - below a bridge in Biedrusko - fertile alder forest Ribo nigriAlnetum and Populetum albae riparian woodland

31. Chludowska Road - acidophilous oak woodland Calamagrostio-Quercetum petraeae

32. Chludowska Road - trees, shrubs and herbaceous plants along forest road

33. Chludowska Road - acidophilous oak woodland Calamagrostio-Quercetum petraeae 
34. Biedrusko-Radojewo road - sub-xerothermophilous oak woodland Potentillo albaeQuercetum

35. Biedrusko-Radojewo road - thermophilous forest edge communities of the class Trifolio-Geranietea sanguinei and oak greenwood

36. Stalina Hill and areas situated to the north - a mixture of grasslands, meadows, thickets and forests

37. the Sarnia Meadow - herb vegetation of the class Molinio-Arrhenatheretea, willow and alder thickets

38. the Sarnia Meadow - semi-moist purple moor-grass meadow Molinietum medioeuropaeum

39. the Sarnia Meadow - blackthorn and hawthorn shrubs Pruno-Crataegetum

40. the Sarnia Meadow - semi-moist purple moor-grass meadow Molinietum medioeuropaeum

41. the Sarnia Meadow - a mixture of grasslands, meadows, shrubs and ash-alder forest

42. a mixture of grasslands, meadows and thickets to the south of Chludowska Road

43. plant community with Almus incana

44. pinewood of the allion Dicrano-Pinion

45. elm-ash forest Ficario-Ulmetum campestris

46. forest road

47. Zlotniki-Biedrusko road - roadside trees and thickets of the class Rhamno-Prunetea

\section{MATERIAL AND METHOD}

The field study was carried out in the years 1997-2008, from early spring to late autumn, at 47 localities representing plant communities most characteristic of this area. In the years $2002-$ 2008 the study was less intense and concentrated on the search for the species not evidenced in the first period of observations. The study was qualitative in character and all known methods of detection of the leaf-mining moths were applied. The main method was the search for mined leaves, both left empty and with larvae. Larvae of later stages, feeding on the surface of the leaves or under a folded leaf margin or lobe, as well as Coleophoridae cases and Bucculatricidae pupal cocoons were also collected.

The mines were usually identified in fresh state. The mined leaves were dried in the same way as for herbarial purposes and placed in labelled paper envelopes. The preimaginal stages were reared taking into regard the recommendations of Borkowski (1969b), Buszko (1990a) and Beiger (1991).

Apart from collection of mined leaves adult specimens were also collected by sweeping with entomological net over the herbaceous vegetation, shaking branches to dislodge them and picking from the tree trunks. The moths were also collected at light. The caught or reared imagines were killed with the vapour of ethyl acetate and set according to the procedures accepted for the Microlepidoptera (Borkowski 1969b; Landry \& Landry 1994). Identification of some species was possible only after careful examination of genital apparatus morphology. Preparation of genital organs was made taking into regard the procedures recommended by Razowski (1973). The nomenclature of Lepidoptera was accepted after Fauna Europaea (2010), and the systematic arrangement of families, genera and species was assumed after a distributional checklist by Buszko \& Nowacki (2000).

\section{RESULTS}

The number of species of leaf-mining moths found over the area of the Biedrusko military area is 258 . They represent 20 families, the most abundantly represented were Gracillariidae (71 species), Nepticulidae (56 species), Coleophoridae (47) and Elachistidae (25). The other families were represented by a bit over ten species (Bucculatricidae - 12 species) or usually by 
a few species. The disproportion follows from the biology of the species belonging to a given family, e.g. in some families only a few species are leaf-miners (Crambidae) and can also be a consequence of a small number of species in a given family, e.g. Tischeriidae. The list of species found in particular types of vegetation together with the time of appearance of different developmental stages and feeding marks are given in Table 1. The last but one column gives the results of the search in the habitats not listed in the table, such as forest edge communities, young tree stands, forest tracts, combinations of plant species difficult for classification, species aggregations etc. (the details are given in the list of study sites). The greatest number of mining moths (164) was collected in those habitats. Large numbers of species were found in oak forests (73), synanthropic communities (64), riparian forests (45), xerothermic grasslands (42) and alder forests (40). Fauna of the pine forest (3 species), heath land (4 species), water and rush communities ( 8 species) was the poorest. Among the species identified, 24 were found at more than 10 sites (Table 1 , entry $8,13,19,22,29,34,37,38,42,59,67,82,93,98$, $106,108,119,139,161,169,187,188,240$ ), including a polyphagous Lyonetia clerkella reported from 24 sites. As many as 66 species were found at single sites.

Twenty-four species of leaf-mining moths were reported for the first time from Greater Poland Voivodeship. Some of them were reported in a distributional checklist by Buszko \& Nowacki (2000) on the basis of my unpublished data. However, these authors gave only the information on the presence of particular species in particular voivodeships (administration districts in Poland), with no data on the sites of collection.

\section{REVIEW OF THE RARE SPECIES \\ NEWLY FOUND TO GREATER POLAND}

\section{Trifurcula cryptella (Stainton, 1856)}

XU 22 (25): xerothermic grassland: 21 IX 1997, several empty mines; 3 VIII 1998, numerous tenanted mines. Host plant: Coronilla varia $\mathrm{L}$.

In Poland, the species so far has been recorded from the Beskid Śląski Mts. (Toll 1947a, b), Sudety Mts. (Borkowski 1969a), Świętokrzyskie Mts. (Michalska 1988) and voivodeships: Lublin and Lesser Poland (Buszko 2000a)

\section{Ectoedemia angulifasciella (Stainton, 1849)}

XU 22 (41): thickets, 26 X 1996, several empty mines; XU 31 (10): 26 X 1997, several empty mines and mines with larvae. Host plant: Rosa canina L. Mines were found on well insolated shrubs.

The species previously reported from Beskid Śląski Mts. (Toll 1947a, 1950), Bielinek Reserve (Buszko \& Baraniak 1989), Ojców National Park (Buszko 1993) and Lower Silesia Voivodeship (Buszko 2000a).

\section{Bucculatrix humiliella Herrich-Schäffer, 1855}

XU 22 (25): semi-moist purple moor-grass meadow, 29 III 1998, one specimen.

Apart from the above record, this very rare species has been found in Poland also in Czarny Dunajec, Nowy Sącz, Kraków (Schille 1931) and Glińsk (Mleczak 2004).

Bucculatrix cristatella (Zeller, 1839)

XU 31 (6), XU 21 (14): xerothermic grasslands and XU 22 (25), XU32 (27): meadows, IV$\mathrm{V}$ and 3/VI-VII externally feeding larvae and white pupal cocoons. Host plant: Achillea millefolium $\mathrm{L}$. 
Table 1. The leaf-mining species found in particular types of vegetation over the area of the military grounds in Biedrusko, in the years $1997-2008$. Abbreviations: * species newly found in Greater Poland Voivodeship, em - empty mine, i - imago, c - cocoon, 1 - larva, p - pupa, 3/V - decade/month, V - month (1-3 decade)

\begin{tabular}{|c|c|c|c|c|c|c|c|c|c|c|c|c|c|c|c|c|c|c|}
\hline No. & Species & $\begin{array}{l}\text { Time of } \\
\text { appearance }\end{array}$ & 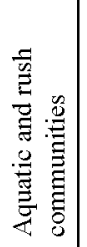 & 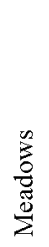 & 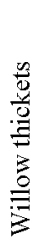 & $\begin{array}{l}\frac{2}{0} \\
\frac{2}{0} \\
\frac{0}{0} \\
\frac{z}{2}\end{array}$ & 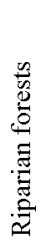 & 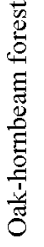 & $\begin{array}{l}\frac{n}{2} \\
\frac{2}{0} \\
\frac{0}{4} \\
\frac{4}{5}\end{array}$ & $\frac{\square}{0}$ & 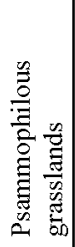 & 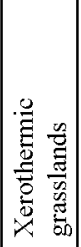 & 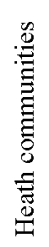 & 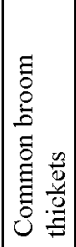 & 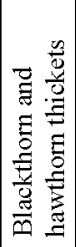 & 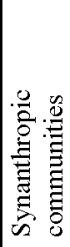 & 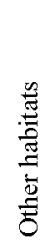 & Study sites \\
\hline 1 & 2 & 3 & 4 & 5 & 6 & 7 & 8 & 9 & 10 & 11 & 12 & 13 & 14 & 15 & 16 & 17 & 18 & 19 \\
\hline \multicolumn{19}{|c|}{ Eriocraniidae } \\
\hline 1. & $\begin{array}{l}\text { Dyseriocrania subpurpurella } \\
\text { (Haw.) }\end{array}$ & $\begin{array}{l}3 / \mathrm{V}-1 / \mathrm{VI} \\
\mathrm{V}\end{array}$ & & & & & & & $\begin{array}{l}1 \\
\mathrm{i}\end{array}$ & & & & & & & & $\begin{array}{l}1 \\
\mathrm{i}\end{array}$ & $10,24,34,36$ \\
\hline 2. & $\begin{array}{l}\text { Heringocrania unimaculella } \\
\text { (Zett.) }\end{array}$ & 3/III-IV & & & & & & & & & & & & & & & $\mathrm{i}$ & 18,25 \\
\hline 3. & $\begin{array}{l}\text { Eriocrania semipurpurella } \\
\text { (Steph.) }\end{array}$ & $\mathrm{V}$ & & & & & & & & & & & & & & & $\mathrm{i}$ & 36 \\
\hline \multicolumn{19}{|c|}{ Nepticulidae } \\
\hline 4. & Enteucha acetosae (Stt.) & VI, VII, IX & & & & & & & & & & 1 & & & & 1 & 1 & $6,28,29,36,41,47$ \\
\hline 5. & \begin{tabular}{|l|}
$\begin{array}{l}\text { Stigmella confusella (Wood et } \\
\text { Wals.) }\end{array}$ \\
\end{tabular} & 24 VI 1998 & & & & & & & & & & & & & & & em & 35 \\
\hline 6. & Stigmella freyella (Heyd.) & VII & & & & & & & & & & & & & & 1 & & 29 \\
\hline 7. & Stigmella tiliae (Frey) & VII, IX-X & & & & & & 1 & 1 & & & & & & & 1 & & $1,12,29,34$ \\
\hline 8. & Stigmella betulicola (Stt.) & $\begin{array}{l}3 / \text { VI-VII, } \\
\text { IX-X }\end{array}$ & & & & 1 & & & 1 & & & & 1 & 1 & & 1 & 1 & $\begin{array}{l}2,5,7,15,18,22,24, \\
28,29,31,32,33,34, \\
35,36,41,42,46,47\end{array}$ \\
\hline 9. & Stigmella luteella (Stt.) & VI, X & & & & & & & $\mathrm{em}$ & & & & & & & & em & $7,33,36$ \\
\hline 10. & Stigmella glutinosae (Stt.) & VII, IX-X & & & & 1 & 1 & & & & & & & & & & & $3,11,13,15$ \\
\hline 11. & Stigmella alnetella (Stt.) & IX & & & & 1 & 1 & & & & & & & & & & & $3,11,15$ \\
\hline 12. & Stigmella microtheriella $(\mathrm{Stt})$. & VII, IX-X & & & & & 1 & 1 & 1 & & & & & & & & 1 & $3,12,32,34$ \\
\hline 13. & Stigmella prunetorum $(\mathrm{Stt})$. & $\begin{array}{l}\text { 3/VI-VII, } \\
\mathrm{IX}-\mathrm{X}\end{array}$ & & & & & & & 1 & & & 1 & & & 1 & 1 & 1 & $\begin{array}{l}1,5,9,10,13,19,20, \\
24,28,29,32,34,36, \\
39,41,42,47\end{array}$ \\
\hline 14. & Stigmella aceris (Frey) & VI, IX & & & & & 1 & & 1 & & & & & & & 1 & 1 & $\begin{array}{l}1,3,9,24,28,29,32, \\
34,47\end{array}$ \\
\hline
\end{tabular}




\begin{tabular}{|c|c|c|c|c|c|c|c|c|c|c|c|c|c|c|c|c|c|c|}
\hline 1 & 2 & 3 & 4 & 5 & 6 & 7 & 8 & 9 & 10 & 11 & 12 & 13 & 14 & 15 & 16 & 17 & 18 & 19 \\
\hline 15. & Stigmella malella (Stt.) & VI, IX-X & & & & & & & & & & & & & & 1 & 1 & $5,10,24$ \\
\hline 16. & Stigmella catharticella (Stt.) & $\begin{array}{l}3 / \mathrm{VI}-\mathrm{VII}, \\
\mathrm{IX}-\mathrm{X}\end{array}$ & & & & & & & & & & & & & & & $\begin{array}{l}1, \\
\text { em }\end{array}$ & $28,30,35$ \\
\hline 17. & Stigmella anomalella (Goeze) & $\mathrm{IX}-\mathrm{X}$ & & & & & & & & & & & & & & 1 & 1 & $9,28,47$ \\
\hline 18. & Stigmella ulmivora (Fol.) & VI, IX-X & & $\checkmark$ & & & 1 & & & & & & & & & & & 3,45 \\
\hline 19. & Stigmella crataegella (Klim.) & VII & & 1 & & & & & 1 & & & & & & 1 & 1 & 1 & $\begin{array}{l}9,20,23,24,28,34, \\
38,39,40,41,47\end{array}$ \\
\hline 20. & Stigmella magdalenae (Klim.) & VII & & & & 1 & 1 & & 1 & & & & & & & & 1 & $3,11,30,33,34$ \\
\hline 21. & Stigmella nylandriella (Tgstr.) & VII & & & & 1 & 1 & & 1 & & & & & & & & 1 & $3,11,24,31,33,34$ \\
\hline 22. & Stigmella oxyacanthella (Stt.) & $\mathrm{IX}-\mathrm{X}$ & & 1 & & & 1 & & & & & & & & 1 & 1 & 1 & $\begin{array}{l}10,20,23,24,28,30 \\
32,34,38,39,40,47\end{array}$ \\
\hline 23. & Stigmella pyri (Glitz) & VIII-2/IX & & 1 & & & & & 1 & & & & & & 1 & 1 & 1 & $\begin{array}{l}9,20,23,29,34,39 \\
40,41\end{array}$ \\
\hline 24. & Stigmella minusculella (H.-S.) & $\begin{array}{l}\text { VI-1/VII, } \\
\text { IX }\end{array}$ & & 1 & & & & & & & & & & & 1 & 1 & 1 & $\begin{array}{l}24,29,30,32,39,40, \\
41,47\end{array}$ \\
\hline 25. & Stigmella hybnerella (Hbn.) & VI, VIII-IX & & 1 & & & & & 1 & & & & & & 1 & 1 & 1 & $\begin{array}{l}10,20,23,24,28,39, \\
40,47\end{array}$ \\
\hline 26. & Sitgmella floslactella (Haw.) & $\begin{array}{l}3 / \mathrm{VI}-2 / \mathrm{VII}, \\
\mathrm{IX}-2 / \mathrm{X}\end{array}$ & & & & & 1 & & 1 & & & & & & & & 1 & $3,24,32,34$ \\
\hline 28. & Stigmella tityrella $(\mathrm{Stt})$. & VII, IX & & & & & & & & & & & & & & & 1 & 24 \\
\hline 29. & Stigmella salicis (Stt.) & $\begin{array}{l}\text { 3/VI-VII, } \\
\text { IX-X }\end{array}$ & & 1 & 1 & 1 & & & & & & & & & & & 1 & $\begin{array}{l}10,11,15,16,21,25, \\
27,28,37,40,41,47\end{array}$ \\
\hline 30. & Stigmella myrtillella (Stt.) & VI, IX & & & & & & & 1 & & & & & & & & & 33 \\
\hline 31. & Stigmella obliquella (Hein.) & VII, IX-X & & & 1 & & 1 & & & & & & & & & 1 & & $7,10,27,30,47$ \\
\hline 32. & Stigmella trimaculella (Haw.) & VII, IX-X & & & & & & & & & & & & & & 1 & 1 & 28,29 \\
\hline 33. & Stigmella assimilella (Zell.) & $\mathrm{IX}$ & & & & & & & & & & & & & & & 1 & 36 \\
\hline 34. & Stigmella plagicolella (Stt.) & VI, IX-X & & & & & & & 1 & & & 1 & & & 1 & 1 & 1 & $\begin{array}{l}1,5,6,9,13,19,20,24 \\
28,29,32,34,36,39,47\end{array}$ \\
\hline 35. & Stigmella lemniscella (Zell.) & VI, IX-X & & & & & 1 & & & & & & & & & 1 & & $3,29,45$ \\
\hline 36. & Stigmella continuella (Stt.) & $\begin{array}{l}16-17 X \\
1998\end{array}$ & & & & em & & & & & & & & & & em & & 15,29 \\
\hline
\end{tabular}




\begin{tabular}{|c|c|c|c|c|c|c|c|c|c|c|c|c|c|c|c|c|c|c|}
\hline 1 & 2 & 3 & 4 & 5 & 6 & 7 & 8 & 9 & 10 & 11 & 12 & 13 & 14 & 15 & 16 & 17 & 18 & 19 \\
\hline 37. & $\begin{array}{l}\text { Stigmella splendidissimella } \\
\text { (H.-S.) }\end{array}$ & $\begin{array}{l}\text { 3/VI-VII, } \\
\text { IX-X }\end{array}$ & & & & 1 & 1 & 1 & 1 & & & & & & & 1 & 1 & $\begin{array}{l}3,9,10,11,12,22,24 \\
28,30,32,34,35,41 \\
46,47\end{array}$ \\
\hline 38. & Stigmella aeneofasciella (H.-S.) & $\mathrm{X}$ & & & & & & & & & & 1 & & 1 & 1 & 1 & 1 & $\begin{array}{l}6,9,10,13,14,19,20, \\
22,23,28,29,35,36,41\end{array}$ \\
\hline 39. & Stigmella incognitella (H.-S.) & $17 \times 1998$ & & & & & & & & & & & & & & 1 & & 24 \\
\hline 40. & Stigmella hemargyrella (Koll.) & $\begin{array}{l}\text { VI, 3/VIII- } \\
\text { IX }\end{array}$ & & & & & & & & & & & & & & & 1 & 24 \\
\hline 41. & Stigmella speciosa (Frey) & VI, VIII & & & & & & 1 & & & & & & & & & 1 & $12,24,32$ \\
\hline 42. & Stigmella basiguttella (Hein.) & VI, IX-X & & & & & 1 & & 1 & & & & & & & & 1 & $\begin{array}{l}10,24,28,31,32,33, \\
34,35,36,45,47\end{array}$ \\
\hline 43. & Stigmella ruficapitella (Haw.) & VI, IX & & & & & 1 & & 1 & & & & & & & & & $33,34,45$ \\
\hline 44. & Stigmella roborella (Joh.) & $3 / \mathrm{VI}-2 / \mathrm{VII}$ & & & & & & & 1 & & & & & & & & & 34 \\
\hline 45. & *Trifurcula cryptella (Stt.) & $\begin{array}{l}21 \text { IX } 1997 \\
3 \text { VIII } 1998 \\
\end{array}$ & & & & & & & & & & $\begin{array}{c}\mathrm{em} \\
1 \\
\end{array}$ & & & & & & 25 \\
\hline 46. & Bohemannia pulverosella (Stt.) & VI-1/VII & & & & & & & & & & & & & & 1 & & 10,24 \\
\hline 47. & Ectoedemia sericopeza (Zell.) & $\mathrm{V}$ & & & & & & & i & & & & & & & i & & 1,34 \\
\hline 48. & Ectoedemia weaveri (Stt.) & 11 III 2001 & & & & & & & & 1 & & & & & & & & 44 \\
\hline 49. & Ectoedemia septembrella (Stt.) & VII, IX & & & & & & & 1 & & & 1 & & & 1 & & 1 & $\begin{array}{l}6,20,23,28,34,35 \\
36,39,41\end{array}$ \\
\hline 50. & Ectoedemia intimella (Zell.) & $3 / \mathrm{IX}-\mathrm{X}$ & & & 1 & 1 & & & & & & & & & & & & $11,15,16,21,25,27$ \\
\hline 51. & Ectoedemia hannoverella (Glitz) & IX-XI & & & & & & & & & & & & & & 1 & 1 & 28,29 \\
\hline 52. & Ectoedemia turbidella (Zell.) & $\mathrm{IX}-\mathrm{X}$ & & & & & 1 & & & & & & & & & 1 & 1 & $29,30,32$ \\
\hline 53. & Ectoedemia argyropeza (Zell.) & $\mathrm{IX}-\mathrm{XI}$ & & & & & & & & & & & & & & & 1 & $28,32,36,41$ \\
\hline 54. & Ectoedemia albifasciella (Hein.) & 3/VIII-IX & & & & & & & 1 & & & & & & & & 1 & $\begin{array}{l}10,24,28,31,32,33 \\
34,36,47\end{array}$ \\
\hline 55. & $\begin{array}{l}\text { Ectoedemia subbimaculella } \\
\text { (Haw.) }\end{array}$ & $\mathrm{IX}-\mathrm{X}$ & & & & & & & 1 & & & & & & & & 1 & $24,31,32,33,34$ \\
\hline 56. & $\begin{array}{l}\text { *Ectoedemia angulifasciella } \\
\text { (Stt.) }\end{array}$ & $\begin{array}{l}26 \times 1996 \\
26 \times 1997\end{array}$ & & & & & & & & & & & & & & & $\begin{array}{l}1, \\
\mathrm{em}\end{array}$ & 10,41 \\
\hline 57. & Ectoedemia atricollis (Stt.) & $\mathrm{IX}$ & & 1 & & & & & 1 & & & & & & 1 & & 1 & $10,24,28,34,39,40$ \\
\hline 58. & Ectoedemia rubivora (Wck.) & $\mathrm{IX}-\mathrm{X}$ & & & & & & & & & & & & & & 1 & 1 & $\begin{array}{l}10,24,28,29,32,41 \\
46,47\end{array}$ \\
\hline
\end{tabular}




\begin{tabular}{|c|c|c|c|c|c|c|c|c|c|c|c|c|c|c|c|c|c|c|}
\hline 1 & 2 & 3 & 4 & 5 & 6 & 7 & 8 & 9 & 10 & 11 & 12 & 13 & 14 & 15 & 16 & 17 & 18 & 19 \\
\hline 59. & Ectoedemia occultella (L.) & $\mathrm{IX}-\mathrm{X}$ & & & & & & & & & & & & & & 1 & 1 & $\begin{array}{l}2,5,7,10,18,24,28, \\
29,31,32,33,34,35, \\
36,41,46,47\end{array}$ \\
\hline \multicolumn{19}{|c|}{ Heliozelidae } \\
\hline 60. & $\begin{array}{l}\text { Antispila metallella (Den. et } \\
\text { Schiff.) }\end{array}$ & \begin{tabular}{|l} 
VI \\
27 IV 1998 \\
\end{tabular} & & & & & & 1 & & & & & & & & & i & $12,32,37$ \\
\hline 61. & Heliozela sericiella (Haw.) & $1 / \mathrm{IX}$ & & & & & & & $\mathrm{em}$ & & & & & & & & & 33,34 \\
\hline 62. & Heliozela resplendella (Stt.) & VII, X & & & & $\mathrm{em}$ & & & & & & & & & & & & $11,13,15$ \\
\hline 63. & Heliozela hammoniella Sorh. & $3 / \mathrm{IX}-2 / \mathrm{X}$ & & & & $\mathrm{em}$ & & & & & & & & & & & $\mathrm{em}$ & 7,15 \\
\hline \multicolumn{19}{|c|}{ Incurvariidae } \\
\hline 64. & Incurvaria pectinea Haw. & VII & & & & & & & & & & & & & & & em & 7,18 \\
\hline 65. & $\begin{array}{l}\text { Incurvaria masculella (Den. et } \\
\text { Schiff.) }\end{array}$ & $\mathrm{V}$ & & & & & & $\mathrm{i}$ & & & & & & & & & & 12 \\
\hline 66. & Phylloporia bistrigella (Haw.) & \begin{tabular}{|l|}
$2 / \mathrm{VII}$ \\
$2 / \mathrm{VII}, 1 / \mathrm{IX}$ \\
\end{tabular} & & & & $\begin{array}{c}1 \\
\mathrm{em}\end{array}$ & & & & & & & $\mathrm{em}$ & & & & em & $15,18,47$ \\
\hline \multicolumn{19}{|c|}{ Tischeriidae } \\
\hline 67. & Tischeria ekebladella (Bjerk.) & VII-IX & & & & & 1 & & 1 & & & & & & & & 1 & $\begin{array}{l}5,10,24,28,31,32,33, \\
34,35,36,41,45,46,47\end{array}$ \\
\hline 70. & Coptotriche marginea (Haw.) & $\mathrm{IX}-\mathrm{X}$ & & & & & & & 1 & & & & & & & & 1 & $28,32,34,47$ \\
\hline 71. & Coptotriche heinemanni (Wck.) & IX $-\mathrm{X}$ & & & & & & & & & & & & & & & 1 & $28,32,46$ \\
\hline 72. & $\begin{array}{l}\text { Coptotriche angusticolella } \\
\text { (Dup.) }\end{array}$ & IX-X & & & & & & & & & & & & & & 1 & 1 & $\begin{array}{l}9,10,24,27,28,29 \\
37,47\end{array}$ \\
\hline \multicolumn{19}{|c|}{ Roeslerstammiidae } \\
\hline 73. & Roeslerstammia erxlebella $(\mathrm{F})$. & VII & & & & & & $\mathrm{i}$ & & & & & & & & & & 12 \\
\hline \multicolumn{19}{|c|}{ Bucculatricidae } \\
\hline 74. & Bucculatrix nigricomella (Zell.) & \begin{tabular}{|l|} 
III-1/V \\
$3 /$ VI-VII \\
\end{tabular} & & 1 & & & & & & & & & & & & & & 41 \\
\hline 75. & Bucculatrix gnaphaliella (Treit.) & $\begin{array}{l}\mathrm{V} \\
\mathrm{VI}\end{array}$ & & & & & & & & & 1 & & & & & & & $4,7,8,17$ \\
\hline 76. & Bucculatrix artemisiella $\mathrm{H}$--S. & \begin{tabular}{|l|} 
IV-V, 3/VI-- \\
VII
\end{tabular} & & & & & & & & & 1 & 1 & & & & & & $4,7,8,10,14,17,19$ \\
\hline 77. & Bucculatrix noltei Petry & $\begin{array}{l}3 / \text { VI-VII, LX } \\
\text { VIII }\end{array}$ & & & & & & & & & & & & & & 1 & i & $\begin{array}{l}5,9,10,13,28,29,32, \\
35,47\end{array}$ \\
\hline
\end{tabular}




\begin{tabular}{|c|c|c|c|c|c|c|c|c|c|c|c|c|c|c|c|c|c|c|}
\hline 1 & 2 & 3 & 4 & 5 & 6 & 7 & 8 & 9 & 10 & 11 & 12 & 13 & 14 & 15 & 16 & 17 & 18 & 19 \\
\hline 78. & *Bucculatrix humiliella $\mathrm{H}$.-S. & 29 III 1998 & & i & & & & & & & & & & & & & & 25 \\
\hline 79. & *Bucculatrix cristatella (Zell.) & $\begin{array}{l}\text { IV-V, 3/VI- } \\
\text { VII }\end{array}$ & & $1, \mathrm{c}$ & & & & & & & & $1, \mathrm{c}$ & & & & & & $6,14,25,27,41$ \\
\hline 80. & Bucculatrix frangutella (Goeze) & $\begin{array}{l}\text { IX } \\
\text { VI }\end{array}$ & & & & & & & $\begin{array}{l}1 \\
\mathrm{i}\end{array}$ & & & & & & & & 1 & $28,32,33,34,35$ \\
\hline 81. & Bucculatrix demaryella (Dup.) & VIII & & & & & & & & & & & & & & & em & 28 \\
\hline 82. & $\begin{array}{l}\text { Bucculatrix bechsteinella } \\
\text { (Bechs. et Scharf.) }\end{array}$ & VI-VII, IX & & 1 & & $\mathrm{em}$ & & & $\begin{array}{c}1, \\
\mathrm{em}\end{array}$ & & & & & & $\begin{array}{c}1, \\
\text { em }\end{array}$ & $\begin{array}{l}1, \\
\text { em }\end{array}$ & $\begin{array}{l}1, \\
\text { em }\end{array}$ & $\begin{array}{l}5,9,10,11,20,23,24, \\
25,28,29,34,38,39, \\
40,47\end{array}$ \\
\hline 83. & Bucculatrix ulmella Zell. & $\begin{array}{l}2 / \mathrm{VI}-1 / \mathrm{VII} \\
\mathrm{IX}\end{array}$ & & & & & & & 1 & & & & & & & & 1 & $\begin{array}{l}10,24,31,32,33,34 \\
46,47\end{array}$ \\
\hline 84. & Bucculatrix cidarella (Zell.) & $\begin{array}{l}\mathrm{IX} \\
\mathrm{X} \\
2 / \mathrm{VII}\end{array}$ & & & & em & & & & & & & & & & & $\begin{array}{c}1 \\
\mathrm{em}\end{array}$ & $7,10,11,15$ \\
\hline 85. & Bucculatrix thoracella (Thnbg.) & $\begin{array}{l}\text { VII, IX } \\
\text { V }\end{array}$ & & & & & & $\begin{array}{l}\mathrm{i} \\
\mathrm{i}\end{array}$ & 1 & & & & & & & 1 & 1 & $\begin{array}{l}1,9,10,12,13,28,30, \\
34\end{array}$ \\
\hline \multicolumn{19}{|c|}{ Gracillariidae } \\
\hline 86. & Parectopa ononidis (Zell.) & V, VII & & & & & & & & & & 1 & & & & & & 6 \\
\hline 87. & *Parectopa robiniella $\mathrm{Clem}$. & 21 IX 1997 & & & & & & & & & & & & & & & em & 32 \\
\hline 88. & Micrurapteryx kollariella (Zell.) & & & $\begin{array}{l}1 \\
\mathrm{i}\end{array}$ & & & & & & & & & & & & & $\mathrm{i}$ & 36,40 \\
\hline 89. & Caloptilia elongella (L.) & $\begin{array}{l}3 / \mathrm{V}-1 / \mathrm{VII}, \\
\mathrm{IX}\end{array}$ & & & & 1 & & & & & & & & & & & & $3,11,13,15$ \\
\hline 90. & Caloptilia betulicola (M. Her.) & $\mathrm{IX}-\mathrm{X}$ & & & & & & & & & & & & & & & $\mathrm{c}$ & $7,28,34,41,47$ \\
\hline 91. & Caloptilia mufipennella (Hbn.) & VI-1/VII & & & & & & & & & & & & & & & $\mathrm{c}$ & $10,24,32$ \\
\hline 92. & Caloptilia alchimiella (Scop.) & V-VI, VIII & & & & & & & $\mathrm{i}$ & & & & & & & & $\mathrm{i}$ & $31,33,34,36$ \\
\hline 93. & Caloptilia stigmatella (F.) & $\begin{array}{l}\text { VIII-X } \\
\text { IV, VI, VIII }\end{array}$ & & $\mathrm{i}$ & $\begin{array}{l}\mathrm{l} \\
\mathrm{i}\end{array}$ & $\mathrm{i}$ & $\mathrm{i}$ & & & & & & & & & & & $\begin{array}{l}3,7,11,21,25,36,37, \\
40,41,42,47\end{array}$ \\
\hline 94. & Caloptilia falconipennella (Hbn.) & 9 VII 1997 & & & & & & & & & & & & & & & $\bar{i}$ & 36 \\
\hline 95. & *Caloptilia fidella (Rtt.) & $\begin{array}{l}\text { IX } 2007- \\
2008\end{array}$ & & & & & & & & & & & & & & & 1 & 28 \\
\hline 96. & Gracillaria syringella $(\mathrm{F})$. & VI, VIII-IX & & & & & 1 & & 1 & & & & & & & 1 & 1 & $1,3,10,29,34$ \\
\hline 97. & $\begin{array}{l}\text { Aspilapteryx tringipennella } \\
\text { (Zell.) }\end{array}$ & VII, IX-X & & & & & & & & & & 1 & & & & & 1 & 6,7 \\
\hline
\end{tabular}




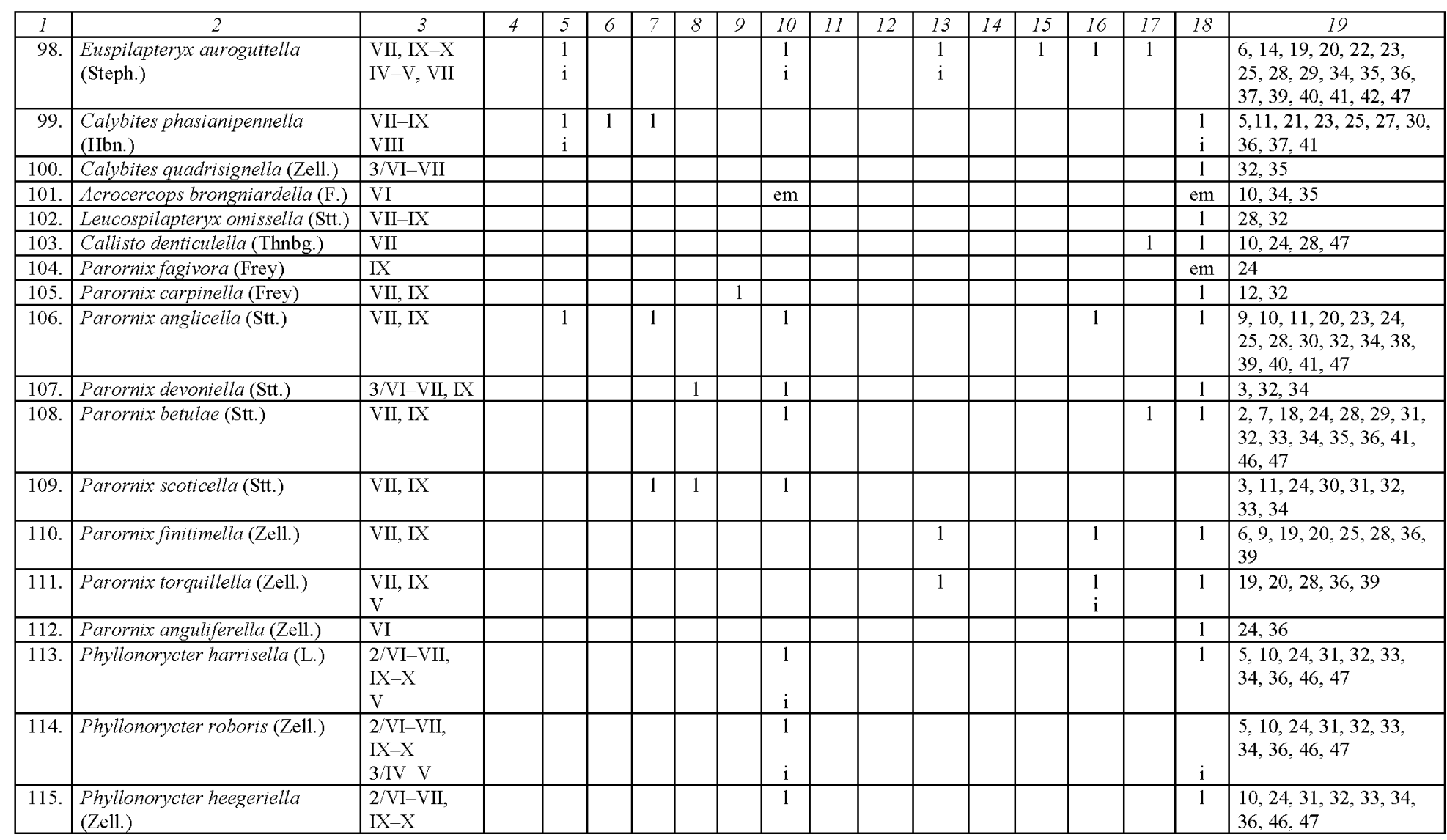




\begin{tabular}{|c|c|c|c|c|c|c|c|c|c|c|c|c|c|c|c|c|c|c|}
\hline 1 & 2 & 3 & 4 & 5 & 6 & 7 & 8 & 9 & 10 & 11 & 12 & 13 & 14 & 15 & 16 & 17 & 18 & 19 \\
\hline 116. & $\begin{array}{l}\text { Phyllonorycter tenerella } \\
\text { (Joann.) }\end{array}$ & VII, IX-X & & & & & & 1 & & & & & & & & & & 12 \\
\hline 117. & $\begin{array}{l}\text { Phyllonorycter kuhlweiniella } \\
\text { (Zell.) }\end{array}$ & $8 \mathrm{~V} 1999$ & & & & & & & i & & & & & & & & & 34 \\
\hline 118. & $\begin{array}{l}\text { Phyllonorycter quercifoliella } \\
\text { (Zell.) }\end{array}$ & $\begin{array}{l}3 / \text { VI-VII, } \\
\text { IX-X } \\
\text { V }\end{array}$ & & & & & & & 1 & & & & & & & & $\mathrm{i}$ & $\begin{array}{l}10,24,31,32,33,34, \\
36,46,47\end{array}$ \\
\hline 119. & $\begin{array}{l}\text { Phyllonorycter oxyacanthae } \\
\text { (Frey) }\end{array}$ & $\begin{array}{l}\text { 2/VI-VII, } \\
\mathrm{IX}-\mathrm{X}\end{array}$ & & 1 & & 1 & & & 1 & & & & & & 1 & & 1 & $\begin{array}{l}9,10,11,20,23,24 \\
28,30,32,34,38,39 \\
40,41,47\end{array}$ \\
\hline 120. & Phyllonorycter sorbi (Frey) & $\begin{array}{l}\text { 3/VI-VII, } \\
\text { IX-X }\end{array}$ & & & & 1 & 1 & & 1 & & & & & & & & & $3,11,30,33,34$ \\
\hline 121. & Phyllonorycter blancardella $(\mathrm{F})$. & VII, IX-X & & & & & & & & & & & & & & 1 & 1 & $10,13,24$ \\
\hline 122. & Phyllonorycter junoniella (Zell.) & 11 III 2001 & & & & & & & & 1 & & & & & & & & 44 \\
\hline 123. & $\begin{array}{l}\text { Phyllonorycter spinicolella } \\
\text { (Zell.) }\end{array}$ & $\begin{array}{l}\text { 3/VI-VII, } \\
\text { IX-X }\end{array}$ & & & & & & & & & & 1 & & & 1 & & 1 & $6,9,13,19,39,41,47$ \\
\hline 124. & $\begin{array}{l}\text { Phyllonorycter lantanella } \\
\text { (Schr.) }\end{array}$ & IX & & & & & & & & & & & & & & & 1 & 9,10 \\
\hline 125 . & Phyllonorycter dubitella (H.-S.) & $\begin{array}{l}\text { 3/VI-VII, } \\
\text { IX-X }\end{array}$ & & & & & & & & & & & & & & & 1 & 28,32 \\
\hline 126. & Phyllonorycter salictella (Zell.) & \begin{tabular}{|l|}
$3 / \mathrm{VI}-\mathrm{VII}$, \\
$\mathrm{IX}-\mathrm{X}$ \\
\end{tabular} & & & 1 & & 1 & & & & & & & & & 1 & 1 & $7,28,29,30,37,47$ \\
\hline 127. & $\begin{array}{l}\text { Phyllonorycter salicicolella } \\
\text { (Sirc.) }\end{array}$ & $\begin{array}{l}\text { 3/VI-VII, } \\
\text { IX-X }\end{array}$ & & 1 & 1 & 1 & & & & & & & & & & & & $\begin{array}{l}11,15,16,21,25,27, \\
30,40\end{array}$ \\
\hline 128. & Phyllonorycter cavella (Zell.) & \begin{tabular}{|l|}
$15-27 \mathrm{X}$ \\
1997
\end{tabular} & & & & & & & & & & & & & & & 1 & 28,47 \\
\hline 129. & $\begin{array}{l}\text { Phyllonorycter maestingella } \\
\text { (Müll.) }\end{array}$ & VII, IX-X & & & & & & & & & & & & & & & 1 & 24 \\
\hline 130. & Phyllonorycter coryli (Nicelli) & $\begin{array}{l}\text { 3/VI-VII, } \\
\text { IX-X }\end{array}$ & & & & & 1 & & 1 & & & & & & & & 1 & $3,32,34$ \\
\hline 131. & $\begin{array}{l}\text { Phyllonorycter esperella } \\
\text { (Goeze) }\end{array}$ & VII, IX & & & & & & 1 & & & & & & & & & & 12 \\
\hline 132. & $\begin{array}{l}\text { Phyllonorycter strigulatella } \\
\text { (Lien. et Zell.) }\end{array}$ & IX-X & & & & & & & & & & & & & & & 1 & 43 \\
\hline 133. & Phyllonorycter rajella $(\mathrm{L})$. & VII, IX-X & & & & 1 & 1 & & & & & & & & & & & $3,10,11,13,15$ \\
\hline
\end{tabular}




\begin{tabular}{|c|c|c|c|c|c|c|c|c|c|c|c|c|c|c|c|c|c|c|}
\hline 1 & 2 & 3 & 4 & 5 & 6 & 7 & 8 & 9 & 10 & 11 & 12 & 13 & 14 & 15 & 16 & 17 & 18 & 19 \\
\hline 134. & $\begin{array}{l}\text { Phyllonorycter nigrescentella } \\
\text { (Logan) }\end{array}$ & VII & & & & & & & 1 & & & & & & & & 1 & 34,35 \\
\hline 135. & $\begin{array}{l}\text { Phyllonorycter medicaginella } \\
\text { (Ger.) }\end{array}$ & $3 / \mathrm{VI}-\mathrm{IX}$ & & & & & & & & & & 1 & & & & 1 & 1 & $6,7,19,28,29$ \\
\hline 136. & Phyllonorycter lautella (Zell.) & VII, IX-X & & & & & & & 1 & & & & & & & & 1 & $10,24,33,34,35,46$ \\
\hline 137. & $\begin{array}{l}\text { *Phyllonorycter robiniella } \\
\text { (Clem.) }\end{array}$ & $\begin{array}{l}\text { VII, IX, X } \\
2002-2008\end{array}$ & & & & & & & & & & & & & & & $1, \mathrm{p}$ & $1,28,29$ \\
\hline 138. & Phyllonorycter schreberella (F.) & $\begin{array}{l}\text { 3/VI-VII, } \\
\text { IX-X }\end{array}$ & & & & & 1 & & & & & & & & & & & 3,45 \\
\hline 139. & $\begin{array}{l}\text { Phyllonorycter ulmifoliella } \\
\text { (Hbn.) }\end{array}$ & VII, IX-X & & & & 1 & & & 1 & & & & & 1 & & 1 & 1 & $\begin{array}{l}2,7,15,18,22,24,28, \\
29,32,33,34,35,36, \\
41,42,46,47\end{array}$ \\
\hline 140. & $\begin{array}{l}\text { Phyllonorycter } \\
\text { emberizaepennella (Bouché) }\end{array}$ & $\begin{array}{l}\text { 3/VI-VII, } \\
\text { IX-X }\end{array}$ & & & & & 1 & 1 & 1 & & & & & & & 1 & 1 & $3,12,13,29,30,34$ \\
\hline 141. & Phyllonorycter stettinensis (Nic.) & VII, IX-X & & & & 1 & 1 & & & & & & & & & & 1 & $3,11,13,15,37$ \\
\hline 142. & $\begin{array}{l}\text { Phyllonorycter froelichiella } \\
\text { (Zell.) }\end{array}$ & VII, IX-X & & & & 1 & 1 & & & & & & & & & & 1 & $3,11,13,15,37$ \\
\hline 143. & \begin{tabular}{|l|} 
Phyllonorycter nicellhi $(\mathrm{Stt})$. \\
\end{tabular} & VII, IX-X & & & & & 1 & & 1 & & & & & & & & 1 & $3,32,34$ \\
\hline 144. & Phyllonorycter kleemannella (F.) & VII, IX-X & & & & 1 & 1 & & & & & & & & & & & $3,11,15$ \\
\hline 145. & Phyllonorycter agilella (Zell.) & VII & & & & & & & & & & & & & & 1 & & 29 \\
\hline 146. & Phyllonorycter acerifoliella (Zell.) & VII, IX-X & & & & & 1 & & & & & & & & & 1 & & 29,45 \\
\hline 147. & $\begin{array}{l}\text { Phyllonorycter joannisi (Le } \\
\text { Marchand) }\end{array}$ & $\begin{array}{l}\text { 3/VI-VII, } \\
\text { IX-X } \\
\text { V }\end{array}$ & & & & & 1 & & 1 & & & & & & & 1 & 1 & $1,3,5,9,24,34,47$ \\
\hline 148. & $\begin{array}{l}\text { Phyllonorycter geniculella } \\
\text { (Rag.) }\end{array}$ & $\begin{array}{l}3 / \mathrm{VI}-\mathrm{VII}, \\
\mathrm{IX}-\mathrm{X}\end{array}$ & & & & & & 1 & & & & & & & & & 1 & $12,24,32$ \\
\hline 149. & *Phyllonorycter issikii (Kum.) & $\begin{array}{l}\text { VII, VIII, IX } \\
\text { 2003-2008 }\end{array}$ & & & & & & $1, \mathrm{p}$ & & & & & & & & & $1, \mathrm{p}$ & $1,12,29$ \\
\hline 150. & Phyllonorycter connexella (Zell.) & VII, IX & & & & & & & & & & & & & & 1 & 1 & 28,29 \\
\hline 151. & Phyllonorycter sagitella (Bjerk.) & $\begin{array}{l}\text { 3/VI-VII, } \\
\text { IX }\end{array}$ & & & & & & & 1 & & & & & & & & 1 & $7,24,33,36,41,42$ \\
\hline 152. & Phyllonorycter comparella (Dup.) & VII, IX-X & & & & & 1 & & & & & & & & & 1 & 1 & $29,30,32$ \\
\hline 153. & $\begin{array}{l}\text { Cameraria ohridella Deschka et } \\
\text { Dimić }\end{array}$ & VI, VIII, IX & & & & & & & & & & & & & & 1 & 1 & 29,32 \\
\hline
\end{tabular}




\begin{tabular}{|c|c|c|c|c|c|c|c|c|c|c|c|c|c|c|c|c|c|c|}
\hline 1 & 2 & 3 & 4 & 5 & 6 & 7 & 8 & 9 & 10 & 11 & 12 & 13 & 14 & 15 & 16 & 17 & 18 & 19 \\
\hline 154. & Phyllocnistis saligna (Zell.) & $\begin{array}{l}3 / \mathrm{VI}-\mathrm{IX} \\
\mathrm{X}\end{array}$ & & & $\begin{array}{l}1 \\
\mathrm{p}\end{array}$ & & & & & & & & & & & & 1 & $7,10,28,37,41,42,47$ \\
\hline 155. & Phyllocnistis unipunctella (Steph.) & VII, IX & & & & & & & & & & & & & & 1 & 1 & 28,29 \\
\hline 156. & Phyllocnistis xenia M. Her. & $\begin{array}{l}\text { VI, IX } \\
1 / \text { VII, X }\end{array}$ & & & & & & & & & & & & & & & $\begin{array}{l}1 \\
\mathrm{p}\end{array}$ & 32 \\
\hline \multicolumn{19}{|c|}{ Yponomeutidae } \\
\hline 157. & Scythropia crataegella (L.) & VI & & & & & & & & & & & & & 1 & & & 28,39 \\
\hline 158. & Ocnerostoma piniariella Zell. & III & & & & & & & & & & & & & & & 1 & 36 \\
\hline 159. & Ocnerostoma friesei $\mathrm{Sv}$. & VI & & & & & & & & & & & & & & & 1 & 36 \\
\hline 160. & Atemelia torquatella (Lien. et Zell.) & $\mathrm{X}$ & & & & & & & & & & & & & & & 1 & 7 \\
\hline 161. & Prays fraxinella (Bjerk.) & 26 VI 1998 & & & & & $\mathrm{i}$ & & & & & & & & & & & 3 \\
\hline \multicolumn{19}{|c|}{ Plutellidae } \\
\hline 162. & Plutella xylostella (L.) & $\mathrm{V}-\mathrm{X}$ & & i & & & & & i & & & $\mathrm{i}$ & & & & i & $\mathrm{i}$ & $\begin{array}{l}1,2,5,6,9,10,20,23 \\
28,29,34,36,37,40,41\end{array}$ \\
\hline \multicolumn{19}{|c|}{ Acrolepiidae } \\
\hline 163. & Acrolepia autumnitella Curt. & VI, IX & & & & 1 & & & & & & & & & & & & 11 \\
\hline \multicolumn{19}{|c|}{ Glyphipterigidae } \\
\hline 164. & Glyphipterix equitella (Scop.) & $\begin{array}{l}\mathrm{V} \\
\mathrm{V}, \mathrm{VIII}\end{array}$ & & & & & & & & & i & & & & & & & 4,8 \\
\hline \multicolumn{19}{|c|}{ Lyonetiidae } \\
\hline 165. & Leucoptera lotella (Hbn.) & VII-IX & & 1 & 1 & & & & & & & & & & & & & 21 \\
\hline 166. & Leucoptera lustratella (H.-S.) & $\begin{array}{l}\text { VI-VII, IX- } \\
\mathrm{X}\end{array}$ & & & & & & & & & & 1 & & 1 & 1 & 1 & 1 & $\begin{array}{l}6,19,20,22,25,29 \\
39,41,47\end{array}$ \\
\hline 167. & Leucoptera laburnella (Stt.) & VI-X & & 1 & & & & & 1 & & & & & & & & & $25,34,36,40,41,42$ \\
\hline 168. & Leucoptera spartifoliella (Hbn.) & $\mathrm{V}$ & & & & & & & & & $\mathrm{c}$ & & & $\mathrm{c}$ & & & & $8,20,22$ \\
\hline 169. & $\begin{array}{l}\text { Leucoptera malifoliella }(\mathrm{O} . \\
\text { Costa) }\end{array}$ & VI-IX & & & & & & & & & & & & & & 1 & 1 & $10,13,24$ \\
\hline 170. & Lyonetia clerkella (L.) & $\begin{array}{l}\text { V, VII, IX- } \\
X\end{array}$ & & 1 & & 1 & 1 & & 1 & & & 1 & & 1 & 1 & 1 & 1 & $\begin{array}{l}1,3,5,7,9,10,11,15 \\
18,20,22,23,24,28 \\
29,30,32,33,34,38 \\
39,40,41,47\end{array}$ \\
\hline 171. & Lyonetia prunifoliella (Hbn.) & VIII-IX & & & & & & & & & & & 1 & & & & 1 & $18,32,47$ \\
\hline \multicolumn{19}{|c|}{ Elachistidae } \\
\hline 172. & Perittia farinella (Thnbg.) & $19 \mathrm{~V} 1997$ & & $\mathrm{i}$ & & & & & & & & & & & & & & 42 \\
\hline 173. & *Perittia herrichiella $(\mathrm{H} .-\mathrm{S})$. & $3 / \mathrm{VI}-\mathrm{VII}$ & & & & & 1 & 1 & 1 & & & & & & & & 1 & $3,12,32,34$ \\
\hline
\end{tabular}




\begin{tabular}{|c|c|c|c|c|c|c|c|c|c|c|c|c|c|c|c|c|c|c|}
\hline 1 & 2 & 3 & 4 & 5 & 6 & 7 & 8 & 9 & 10 & 11 & 12 & 13 & 14 & 15 & 16 & 17 & 18 & 19 \\
\hline 174. & *Stephensia brunnichella (L.) & VI & & & & & & & 1 & & & & & & & & & 34 \\
\hline 175. & Elachista freyerella (Hbn.) & \begin{tabular}{|l|} 
IV $-V$ \\
V \\
\end{tabular} & & $\mathrm{i}$ & & 1 & & & & & & & & & & & 1 & $11,27,32$ \\
\hline 176. & Elachista utonella Frey & \begin{tabular}{|l|}
$\mathrm{IV}-1 / \mathrm{VI}$ \\
$\mathrm{VI}$
\end{tabular} & $\mathrm{i}$ & $\mathrm{i}$ & $\mathrm{i}$ & 1 & & & & & & $\mathrm{i}$ & & & & & & $6,15,21,26,27$ \\
\hline 177. & Elachista poae Stt. & IV & 1 & & & & & & & & & & & & & & & 26 \\
\hline 178. & Elachista alpinella $\mathrm{Stt}$. & V-VII & & & & 1 & & & & & & & & & & & & 15 \\
\hline 179. & *Elachista elegans Frey & $18 \mathrm{~V} 1998$ & & & & & & & 1 & & & & & & & & & 33 \\
\hline 180. & Elachista luticomella Zell. & III-V & & & & & 1 & 1 & & & & & & & & & & 12,45 \\
\hline 181. & Elachista albifrontella (Hbn.) & IV-V & & 1 & & & & & & & & & & & & & 1 & $5,32,38,40,41$ \\
\hline 182. & Elachista apicipunctella $\mathrm{Stt}$. & 1 XII 2000 & & & & & & 1 & & & & & & & & & & 12 \\
\hline 183. & *Elachista subnigrella Dougl. & \begin{tabular}{|l|}
$\mathrm{IV}-1 / \mathrm{V}$ \\
$\mathrm{V}$ \\
\end{tabular} & & 1 & & & & & & & & $\begin{array}{l}1 \\
\mathrm{i} \\
\end{array}$ & & & & & & $6,14,38,40$ \\
\hline 184. & Elachista humilis Zell. & \begin{tabular}{|l|l|}
$1 \mathrm{~V} 1998$ \\
\end{tabular} & & 1 & & & & & & & & & & & & & & 38 \\
\hline 185. & Elachista canapennella (Hbn.) & $\mathrm{V}$ & & $\mathrm{i}$ & & & & & & & & $\mathrm{i}$ & & & & & & $6,38,40,41$ \\
\hline 186. & Elachista anserinella Zell. & \begin{tabular}{|l|}
16 VI 1997, \\
$13-27 \mathrm{~V}$ \\
1998 \\
\end{tabular} & & & & & & & & & & $\overline{\mathrm{i}}$ & & & & & & 6 \\
\hline 188. & Elachista argentella $(\mathrm{Cl})$. & $\begin{array}{l}\mathrm{IV}-\mathrm{V} \\
\mathrm{V}-\mathrm{VI}\end{array}$ & & $\begin{array}{l}1 \\
\mathrm{i}\end{array}$ & $\mathrm{i}$ & & & & $\mathrm{i}$ & & & $\begin{array}{l}1 \\
\mathrm{i}\end{array}$ & & 1 & 1 & & 1 & $\begin{array}{l}5,6,14,19,20,21,22, \\
23,25,27,34,36,38, \\
39,40,41,42\end{array}$ \\
\hline 189. & Elachista pollinariella Zell. & \begin{tabular}{|l|}
$\mathrm{IV}-2 / \mathrm{V}$ \\
$\mathrm{V}-\mathrm{VI}$ \\
\end{tabular} & & 1 & & & & & $\mathrm{i}$ & & & $\begin{array}{l}1 \\
\mathrm{i} \\
\end{array}$ & & 1 & & & $\begin{array}{l}1 \\
\mathrm{i} \\
\end{array}$ & $\begin{array}{l}2,5,6,10,14,22,23 \\
32,34,35,38,40,41 \\
\end{array}$ \\
\hline 190. & Elachista griseella (Dup.) & IV $-1 / \mathrm{V}$ & & & & & & & & & & & & & & & 1 & $10,13,37$ \\
\hline 191. & *Elachista unifasciella (Haw.) & IV & & & & & 1 & 1 & & & & & & & & & & 12,45 \\
\hline 192. & *Elachista gangabella Zell. & 12 IX 2000 & & & & & & & 1 & & & & & & & & & 34 \\
\hline 193. & Elachista adscitella Stt. & $\mathrm{V}-\mathrm{VI}$ & & & & 1 & 1 & & & & & & & & & & & 11,41 \\
\hline 194. & Elachista bisulcella (Dup.) & \begin{tabular}{|l|}
14 VIII 1998 \\
1 VIII 2000 \\
\end{tabular} & & $\begin{array}{l}\mathrm{i} \\
\mathrm{i}\end{array}$ & & & & & & & & & & & & & $\bar{i}$ & $36,40,42$ \\
\hline 195. & Elachista pullicomella Zell. & V, VII & & & & & & & & & $\mathrm{i}$ & $\mathrm{i}$ & & & & & & $6,8,14,19,27$ \\
\hline 196. & *Elachista bedellella (Sirc.) & V, VIII & & $\mathrm{i}$ & & & & & & & $\mathrm{i}$ & $\mathrm{i}$ & & & & & & $6,8,40$ \\
\hline
\end{tabular}




\begin{tabular}{|c|c|c|c|c|c|c|c|c|c|c|c|c|c|c|c|c|c|c|}
\hline 1 & 2 & 3 & 4 & 5 & 6 & 7 & 8 & 9 & 10 & 11 & 12 & 13 & 14 & 15 & 16 & 17 & 18 & 19 \\
\hline \multicolumn{19}{|c|}{ Batrachedridae } \\
\hline 197. & Batrachedra pinicolella (Zell.) & VII & & & & & & & & & & & & & & & $\bar{i}$ & 36 \\
\hline \multicolumn{19}{|c|}{ Coleophoridae } \\
\hline 198. & Coleophora lutipennella (Zell.) & $\begin{array}{l}\mathrm{V}-1 / \mathrm{VI} \\
\mathrm{VII}\end{array}$ & & & & & & & $\begin{array}{l}1 \\
\mathrm{i}\end{array}$ & & & & & & & & $\mathrm{i}$ & $31,33,34,36$ \\
\hline 199. & Coleophora gryphipennella (Hbn.) & $\mathrm{V}$ & & & & & & & & & & & & & & & 1 & 10 \\
\hline 200. & Coleophora flavipennella (Dup.) & $\begin{array}{l}\text { V } \\
\text { VI-VII }\end{array}$ & & & & & & & $\begin{array}{l}1 \\
\mathrm{i}\end{array}$ & & & & & & & & $\mathrm{i}$ & $31,33,34,36$ \\
\hline 201. & Coleophora milvipennis Zell. & VI-VII & & & & i & & $\mathrm{i}$ & & & & & & & & & & 11,12 \\
\hline 202. & Coleophora alnifoliae Barasch & VII & & & & & $\mathrm{i}$ & & & & & & & & & & & 3 \\
\hline 203. & $\begin{array}{l}\text { Coleophora badiipennella } \\
\text { (Dup.) }\end{array}$ & $\begin{array}{l}\text { V } \\
\text { VI }\end{array}$ & & & & & $\mathrm{i}$ & & $\mathrm{i}$ & & & & & & & & & 3,34 \\
\hline 204. & Coleophora serratella $(\mathrm{L})$. & $\begin{array}{l}\text { V } \\
\text { VI-VII }\end{array}$ & & & & $\begin{array}{l}1 \\
\mathrm{i}\end{array}$ & $\begin{array}{l}1 \\
\mathrm{i}\end{array}$ & & 1 & & & & & 1 & & 1 & $\begin{array}{l}\mathrm{i} \\
\mathrm{i}\end{array}$ & $\begin{array}{l}3,11,15,22,29,33, \\
34,41\end{array}$ \\
\hline 205. & Coleophora spinella (Schr.) & $\begin{array}{l}\text { VI } \\
\text { 3/VI-VII }\end{array}$ & & & & & $\mathrm{i}$ & $\mathrm{i}$ & & & & & & & 1 & 1 & $\mathrm{i}$ & $3,12,24,29,36,39$ \\
\hline 206. & Coleophora prunifoliae Doets & IX & & & & & & & & & & & & & 1 & & 1 & 39,41 \\
\hline 207. & Coleophorafuscocuprella $\mathrm{H} .-\mathrm{S}$. & $\mathrm{IX}$ & & & & & & & 1 & & & & & & & & & 34 \\
\hline 208. & $\begin{array}{l}\text { Coleophora lusciniaepennella } \\
\text { (Treit.) }\end{array}$ & $\mathrm{V}, \mathrm{X}$ & & & 1 & 1 & & & & & & & & & & & & $11,15,16,21,25,27$ \\
\hline 209. & Coleophora vitisella Gregs. & 11 IV 1999 & & & & & & & & 1 & & & & & & & & 44 \\
\hline 210. & Coleophora juncicolella Stt. & 25 IV 1999 & & & & & & & & & & & 1 & & & & & 18 \\
\hline 211. & Coleophora orbitella Zell. & $\mathrm{IX}-\mathrm{X}$ & & & & 1 & 1 & & & & & & & & & 1 & & $3,11,13,15,29$ \\
\hline 212. & Coleophora binderella (Koll.) & $\begin{array}{l}\mathrm{V} \\
\mathrm{VI}\end{array}$ & & & & & 1 & & & & & & & & & & i & 3,36 \\
\hline 213. & Coleophora ahenella Hein. & IX & & & & 1 & & 1 & & & & & & & & 1 & 1 & $10,12,24,29$ \\
\hline 214. & Coleophora albitarsella Zell. & $\mathrm{IV}-\mathrm{V}, \mathrm{IX}-\mathrm{X}$ & & & & & & & 1 & & & 1 & & & & & 1 & $6,19,34,35$ \\
\hline 215. & *Coleophora conyzae Zell. & $\mathrm{V}$ & & 1 & & & & & & & & 1 & & & & & & $6,40,41,42$ \\
\hline 216. & Coleophora lineolea (Haw.) & $\begin{array}{l}\mathrm{V}-1 / \mathrm{VI} \\
3 / \mathrm{IX}\end{array}$ & & & & & & & & & & & & & & 1 & 1 & 1,10 \\
\hline 217. & $\begin{array}{l}\text { Coleophora hemerobiella } \\
\text { (Scop.) }\end{array}$ & $\begin{array}{ll}\text { VI } \\
\text { VII }\end{array}$ & & & & & & & & & & & & & 1 & 1 & $\begin{array}{l}1 \\
\mathrm{i}\end{array}$ & $\begin{array}{l}9,20,23,28,29,36 \\
39,47\end{array}$ \\
\hline 218. & Coleophora colutella $(\mathrm{F})$. & $\mathrm{V}-\mathrm{VI}$ & & & & & & & & & & & & 1 & & & 1 & $22,35,41,42$ \\
\hline 219. & Coleophora saturatella $\mathrm{Stt}$. & $1 / \mathrm{VI}$ & & & & & & & & & & & & & & & 1 & 20 \\
\hline
\end{tabular}




\begin{tabular}{|c|c|c|c|c|c|c|c|c|c|c|c|c|c|c|c|c|c|c|}
\hline 1 & 2 & 3 & 4 & 5 & 6 & 7 & 8 & 9 & 10 & 11 & 12 & 13 & 14 & 15 & 16 & 17 & 18 & 19 \\
\hline 220. & Coleophora albicostella (Dup.) & $\mathrm{IV}, \mathrm{X}$ & & & & & & & & & 1 & 1 & & & & & & $6,8,14,19$ \\
\hline 221. & Coleophora discordella Zell. & IX-X & & & & & & & & & & 1 & & & & & & 6 \\
\hline 222. & Coleophora ballotella (F. v R.) & $\mathrm{V}-1 / \mathrm{VI}$ & & & & & & & & & & & & & & 1 & & 1 \\
\hline 223. & $\begin{array}{l}\text { Coleophora albidella (Den. et } \\
\text { Schiff.) }\end{array}$ & $\mathrm{V}$ & & & 1 & & & & & & & & & & & & 1 & 16,32 \\
\hline 224. & Coleophora kuehnella (Goeze) & $\begin{array}{l}\text { VI } \\
\text { VII }\end{array}$ & & & & & & & $\begin{array}{l}\mathrm{l} \\
\mathrm{i}\end{array}$ & & & & & & & & 1 & $31,33,34,35,36$ \\
\hline 225. & Coleophora ibipennella Zell. & $\begin{array}{l}\text { VI } \\
\text { VII }\end{array}$ & & & & & & & $\begin{array}{l}\mathrm{l} \\
\mathrm{i}\end{array}$ & & & & & & & & & $31,33,34,35$ \\
\hline 226. & *Coleophora brevipalpella Wck. & $\mathrm{V}$ & & & & & & & & & & & & & & & 1 & 23 \\
\hline 227. & Coleophora auricella $(\mathrm{F})$. & $\begin{array}{l}\mathrm{IV}-\mathrm{V}, \mathrm{X} \\
\text { VI }\end{array}$ & & $\begin{array}{l}1 \\
\mathrm{i}\end{array}$ & & & & & & & & & & & & & & 38 \\
\hline 228. & Coleophora vibicigerella Zell. & 17 V 1997 & & & & & & & & & 1 & & & & & & & 7 \\
\hline 229. & \begin{tabular}{|l} 
*Coleophora conspicuella Zell. \\
\end{tabular} & $\begin{array}{l}\text { V-1/VI } \\
\text { VII }\end{array}$ & & $\begin{array}{l}1 \\
\mathrm{i}\end{array}$ & & & & & & & & 1 & & & & & $\begin{array}{l}1 \\
\mathrm{i}\end{array}$ & $\begin{array}{l}6,14,20,23,38,40 \\
41,42\end{array}$ \\
\hline 230. & Coleophora fuscociliella Zell. & $\mathrm{V}$ & & & & & & & & & & 1 & & & & 1 & 1 & $19,28,29$ \\
\hline 231. & Coleophora caelebipennella Zell. & $\mathrm{V}$ & & & & & & & & & & 1 & & & & & & 19 \\
\hline 232. & \begin{tabular}{|l|} 
Coleophora vibicella (Hbn.) \\
\end{tabular} & $\begin{array}{l}\text { V-VI } \\
\text { 3/VI-VII }\end{array}$ & & $\begin{array}{l}1 \\
\mathrm{i}\end{array}$ & & & & & & & & 1 & & & & & 1 & $\begin{array}{l}20,23,25,27,36,40, \\
41,42\end{array}$ \\
\hline 233. & *Coleophora lixella Zell. & $\begin{array}{l}\text { IV-V } \\
\text { VI-1/VIII }\end{array}$ & & $\begin{array}{l}1 \\
\mathrm{i}\end{array}$ & & & & & & & & $\begin{array}{l}1 \\
\mathrm{i}\end{array}$ & & & & & 1 & $\begin{array}{l}6,14,22,23,25,40 \\
41,42\end{array}$ \\
\hline 234. & $\begin{array}{l}\text { Coleophora pennella (Den. et } \\
\text { Schiff.) }\end{array}$ & $\mathrm{V}$ & & & & & & & & & & $\mathrm{p}$ & & & & & $\mathrm{p}$ & 7,19 \\
\hline 235. & Coleophora laricella (Hbn.) & $\mathrm{V}$ & & & & & & & & & & & & & & & 1 & 9 \\
\hline 236. & Coleophora gnaphalii Zell. & $\begin{array}{l}\mathrm{V} \\
3 / \mathrm{V}-\mathrm{VI}\end{array}$ & & & & & & & & & $\begin{array}{l}1 \\
\mathrm{i}\end{array}$ & & & & & & & $4,8,17$ \\
\hline 237. & Coleophora galbulipennella Zell. & $1 / \mathrm{VI}$ & & & & & & & & & 1 & & & & & & & 17 \\
\hline 238. & *Coleophora millefolii Zell. & $\begin{array}{l}\mathrm{V}-1 / \mathrm{VI} \\
\mathrm{VI}-\mathrm{VII} \\
\end{array}$ & & & & & & & & & & $\begin{array}{l}1 \\
\mathrm{i}\end{array}$ & & & & & $\mathrm{T}$ & $23,25,35$ \\
\hline 239. & *Coleophora peribenanderi Toll & $\begin{array}{l}\text { VIII-IX } \\
\text { V-VII }\end{array}$ & & & & & & & & & & & & & & & $\begin{array}{l}\mathrm{l} \\
\mathrm{i}\end{array}$ & $28,29,47$ \\
\hline 240. & *Coleophora ramosella Zell. & $3 / \mathrm{IV}-\mathrm{V}$ & & & & & & & & & & 1 & & & & & & 41 \\
\hline 241. & Coleophora trochilella (Dup.) & $\mathrm{V}-1 / \mathrm{VI}$ & & 1 & & & & & & & & 1 & & 1 & 1 & 1 & 1 & $\begin{array}{l}6,9,10,14,20,22,23,25, \\
35,36,38,39,40,41,42\end{array}$ \\
\hline
\end{tabular}




\begin{tabular}{|c|c|c|c|c|c|c|c|c|c|c|c|c|c|c|c|c|c|c|}
\hline 1 & 2 & 3 & 4 & 5 & 6 & 7 & 8 & 9 & 10 & 11 & 12 & 13 & 14 & 15 & 16 & 17 & 18 & 19 \\
\hline 242. & *Coleophora directella Zell. & $\begin{array}{l}3 / \mathrm{IV}-1 / \mathrm{VI} \\
\mathrm{VII}\end{array}$ & & & & & & & & & $\begin{array}{l}1 \\
\mathrm{i}\end{array}$ & 1 & & & & & & $6,8,14,17,19$ \\
\hline 243. & Coleophora expressella Klem. & \begin{tabular}{|l|}
$\mathrm{V}-1 / \mathrm{VI}$ \\
$\mathrm{VI}-\mathrm{VII}$
\end{tabular} & & & & & & & & & & i & & & & & 1 & $23,25,35,41$ \\
\hline 244. & Coleophora saponariella Heeg. & VII-IX & & & & & & & & & & & & & & 1 & 1 & 9,29 \\
\hline \multicolumn{19}{|c|}{ Momphidae } \\
\hline 245. & Mompha langiella (Hbn.) & $\mathrm{VI}-2 / \mathrm{VII}$ & 1 & & 1 & 1 & & & & & & & & & & & & $11,21,26$ \\
\hline 246. & Mompha raschkiella (Zell.) & \begin{tabular}{|l|} 
VI \\
$1 /$ VIII \\
\end{tabular} & & & & & & & & & & & & & & & $\mathrm{i}$ & 36 \\
\hline 247. & $\begin{array}{l}\text { Mompha epilobiella (Den. et } \\
\text { Schiff.) }\end{array}$ & \begin{tabular}{|l|} 
VII \\
VIII
\end{tabular} & 1 & $\mathrm{i}$ & & & & & & & & & & & & & 1 & $26,27,41$ \\
\hline \multicolumn{19}{|c|}{ Cosmopterigidae } \\
\hline 248. & Vulcaniella pomposella (Zell.) & \begin{tabular}{|l|}
4 V 1997 \\
16 VI 1997 \\
\end{tabular} & & & & & & & & & $\begin{array}{l}1 \\
\mathrm{i} \\
\end{array}$ & & & & & & & 4,8 \\
\hline 249. & Cosmopterix zieglerella (Hbn.) & VIII & & & & 1 & & & & & & & & & & & 1 & $10,11,32$ \\
\hline 250. & Cosmopterix scribaiella Zell. & $\mathrm{IX}-\mathrm{X}$ & 1 & & & & & & & & & & & & & & & 16,26 \\
\hline \multicolumn{19}{|c|}{ Gelechiidae } \\
\hline 251. & Chrysoesthia drurella (F.) & $\mathrm{V}$ & & & & & & & & & & & & & & $\mathrm{i}$ & & 29 \\
\hline 254. & $\begin{array}{l}\text { *Syncopacma ochrofasciella } \\
\text { (Toll) }\end{array}$ & VI & & 1 & & & & & & & & & & & & & 1 & $35,40,41$ \\
\hline \multicolumn{19}{|c|}{ Crambidae } \\
\hline 255. & Elophila nympheata (L.) & VIII & $\mathrm{i}$ & & & & & & & & & & & & & & & 26 \\
\hline 256. & Cataclysta lemnata (L.) & 27 V 2002 & $\mathrm{i}$ & & & & & & & & & & & & & & & 26 \\
\hline 257. & Cynaeda dentalis (Den. et Schiff.) & VI-VIII & & & & & & & & & & & & & & & $\mathrm{i}$ & 36 \\
\hline 258. & Epascestria pustulalis (Hbn.) & VI & & & & & & & & & & & & & & & $\mathrm{i}$ & 36 \\
\hline \multicolumn{3}{|c|}{ Total number of recorded species } & 8 & 42 & 15 & 40 & 45 & 22 & 73 & 3 & 12 & 42 & 4 & 13 & 25 & 64 & 164 & \\
\hline
\end{tabular}


The moth previously reported from Lower Silesia and Greater Poland voivodeships, and after 1960 found in Kuyavian-Pomeranian and Lubusz voivodeships (Buszko 2000b). Recently reported from Stary Zalom Reserve (Baraniak \& Walczak 2000) and S-E Poland (Baran 2003).

\section{Parectopa robiniella Clemens, 1863}

XU 32 (32): forest road: 21 IX 1997, several empty mines. Host plant: Robinia pseudacacia L.

This invasive species is native to North America and was accidentally introduced to Europe. For the first time it was found near Milan, northern Italy, in 1970 (Vidano \& Marletto 1971). At present, the species is known from western, southern and central parts of Europe. In Poland, has been so far recorded from S-E part of the country and Kuyavian-Pomeranian Voivodeship ( Buszko 2000c).

\section{Caloptilia fidella (Reutti, 1853)}

XU 32 (28): shrub communities along road, IX 2007 and 2008, empty mines and older larvae under an rolled leaf margin. Host plant: Humulus lupulus L.

The south European species which expanding its range to the north. In Poland, it has been found for the first time in 2003. Recently recorded from Glińsk (Mleczak 2004) and Skarpa Ursynowska Reserve in Warsaw (Jaworski 2009)

\section{Elachista elegans Frey, 1859}

XU 22 (33): acidophilous oak wood, one adult specimen has been reared from tenanted mines collected $18 \mathrm{~V} 1998$ on Calamagrostis arundinacea (L.) Roth.

In Poland, the species has been so far known only from West Pomeranian (Hering 1891) and Warmian-Masurian vivodeships (Buszko 2000d).

\section{Elachista unifasciella (Haworth, 1828)}

XU 21 (12): oak-hornbeam forest, one specimen has been reared from larvae collected 21 IV 1999: XU 31 (45), ash-alder forest, 12 specimens have been reared from larvae collected 15 IV 1999. Host plant: Dactylis glomerata L. subsp. aschersoniana (Graebn.) Thell..

The species has been hitherto reported from Bielinek Reserve (Buszko \& Baraniak 1989), Las Piwnicki Reserve and NE Poland (Buszko 1990b).

\section{Elachista gangabella Zeller, 1850}

XU 32 (34): sub-xerothermophilous oak wood, one specimen has been reared from the larvae collected 12 IX 2000 on Melica nutans L.

In Poland, this elachistid moth has been so far known from West Pomeranian, KuyavianPomeranian, Łódź and Lesser Poland voivodeships (Buszko 2000d).

\section{Coleophora conyzae Zeller, 1868}

XU 31 (6): xerothermic grassland: few cases were found in May 1997-1999 on Inula salicina L.; XU $22(40,41,42)$ : semi-moist purple moor-grass meadows, numerous cases were collected in May 1997-2000 on I. salicina. 20 adult specimens has been reared from the larvae collected $19 \mathrm{~V} 1997$.

The first notice on the occurrence of the species in Poland was mentioned in a genital apparatuses description (Toll 1962). The data refer to two specimens collected 10 VII 1877 in the vicinities of Wroclaw. Microscopic slides of the female and male genitals are deposited in the Humboldt Museum of Berlin (Germany). However, the species was omitted in the monograph on Coleophoridae (Razowski 1990) and in later checklist of the Polish Lepidoptera 
(Rynarzewski 2000). Recently, the moth has been collected in the Brzeźno Reserve (Buszko et al. 1996).

\section{Coleophora brevipalpella Wocke, 1874}

XU 22 (23): herb communities at the edge of small pond: 14 adult specimens have been reared from larvae collected $26 \mathrm{~V} 1997$. Host plant: Serratula tinctoria L.

This very rare species has been so far recorded only from Wroclaw vicinities (Wocke 1874), Kuyavian-Pomeranian and Lubusz voivodeships (Rynarzewski 2000).

\section{Syncopacma ochrofasciella (Toll, 1936)}

XU 22 (40): semi-moist purple moor-grass meadow, XU 22 (41): roadside; XU 32 (35): thermophilous forest edge communities larvae mining the leaves of Astragalus glycyphyllos $\mathrm{L}$. were found in June, July and September on all mentioned localities, at well insolated places. Ten specimens have been reared from larvae collected 30 VI 1998, four from larvae collected 24 VI 1999 and twelve from larvae collected 01 VII 2008.

This very rare species is known only from the Zbocza Phutowskie Reserve (Elsner et al. 1999), where it was collected in 1988. In the monograph on Middle European Gelechiidae (Elsner et al. 1999) the moth has not been included to leaf-miners. Whereas during the studies it was observed, the larva mines the leaves of the host plant. At first it makes small, full depth mines. Then larva spun the leaflets together and continues mining. There is no frass in the mines which is deposited between spun leaflets. The larvae are capable to move to another leaf and start a new mine.

\section{DISCUSSION}

Taking up the studies in the military grounds Biedrusko in 1997, it was assumed that the richness and diversity of the flora in this area would be accompanied by high diversity of leafmining moths. The number of species of such moths found in the area reached 258, which makes $47 \%$ of this fauna reported from Poland. Some of them occurred at very sparse sites, and 24 of the species were reported for the first time from Greater Poland Voivodeship. The species composition presented in this paper should not be treated as final and it is reasonable to expect its enrichment.

The high diversity of leaf-mining species established over the area of the military grounds in Biedrusko is a consequence of a few factors, of which the most important are the richness of the flora and heterogeneity of the space. The diversity of moth species is correlated with the diversity of plants because of the close trophic relation of the moths and the host (the majority of the leaf-mining moths are monophagous or oligophagous). Rich and diverse flora offers the conditions for existence of many species of moths. The area of the military grounds is characterised by mosaic type of vegetation structure developed as a result of deforestation of a large section of the area for the needs of army training. With time over the deforested area some interesting types of semi-natural vegetation developed, including psammophilous grasslands, xerothermic grasslands, meadows (among others the thermophilous Molinia meadows), common broom thickets, shrubs with blackthorn and hawthorn, initial forms of forests, thermophilous forest edge herb communities. These and the other plant communities developed into a mosaic structure of habitats which among other factors determined the species richness of the moth over this area. The spatial mosaic structure of vegetation is one of the factors favourable for preservation and increase in the diversity of the fauna species (Trojan \& Wytwer 1995). The effect of ecotone phenomena manifested at the borders of different habitats must be also of significance. In the thermophilous open communities such as the abovementioned grasslands, Molinia meadows or forest edge communities the fauna, including moths, was very rich. Xerothermic communities were originally related to the sloping banks of 
river valleys and occupied a small percent of the area of the Wielkopolska region (Wojterski et al. 1981). Thus, the moth species living in this type of habitat were probably not numerous. In forest habitats the species richness of fauna has been suggested to depend on the vertical structure of vegetation (Krebs 1996), besides the floristic composition. However, no direct evidence of this influence has been presented.

The results of the study of the Lepidoptera in the area of the military ground in Biedrusko showed the role of the military used areas for protection of species diversity. This phenomenon has been observed in this area for the butterflies (Walczak 2002). Also other military grounds in Europe have been indicated as playing a significant role in protection of the fauna and flora (Toro 1993, IUCN 1995, Klapkarek \& Beutler 1999, Beutler 2000, Eisermann 2000). Because of the character of their use, the military grounds are isolated from some negative forms of human activity related to agriculture, settlements and industry. Nevertheless, the total abandonment of the area can be threatening to some species because of the progressing succession of vegetation. Therefore, it would be recommended to actively protect at least some of the open communities, while preserving the military use of the area.

\section{ACKNOWLEDGEMENTS}

I would like to thank Prof. J. Buszko (Torun, Poland) for confirming my identification of several species. I am indebted to Dr. W. Mey (Berlin, Germany) who kindly loaned slides of Coleophora conyzae deposited in Humboldt Museum in Berlin. I thank also Land Forces Training Centre (Poznań, Poland) who granted me permission for access to the military land. I am especially grateful to Maj. K. Knerek and Col. J. Winko who helped me in many ways. I would also like to thank Dr. H. Beutler (Beeskow, Germany), Prof. J. Borysiak (Poznań, Poland), Prof. Z. Laštüvka (Brno, Czech Republic) and Dr. M. Nuss (Dresden, Germany) for providing some of the literature sources.

\section{REFERENCES}

BARAN T. 2003. Nowe stanowiska rzadkich gatunków Microlepidoptera (Lepidoptera) z miejscowości Tyczyn (południowo-wschodnia Polska). Wiadomości Entomologiczne 22: 117-119.

BARANIAK E. \& WALCZAK U. 2000. Interesujące gatunki motyli (Lepidoptera) z rezerwatu „Stary Zalom”. Badania Fizjograficzne nad Polską Zachodnią, seria C, 47: 45-48.

BARTKOWSKI T. 1962. Próba kartograficznego ujęcia geomorfologii okolic Buka, Szamotuł i Skoków. Prace Komisji Geograficzno-Geologicznej 3: 1-50.

BEIGER M. 1991. Owady minujące. Wydawnictwo Naukowe UAM, Seria Zoologia Nr 17, 155 pp.

BEUTLER H. 2000. Landschaft in neuer Bestimmung. Russische Truppenübungsplätze. Findling, Neuenhagen, 192 pp.

BORKOWSKI A. 1969a. Studien an Stigmelliden (Lepidoptera). Teil I. Zur Verbreitung, Biologie und Ökologie der Stigmelliden in den polnischen Sudeten. Polskie Pismo Entomologiczne 39: 95-122.

BORKOWSKI A. 1969b. O hodowli larw owadów minujących. Polskie Pismo Entomologiczne 39: 185-190.

BORYSIAK J. \& BRZEG A. 1994. Materiały do znajomości szaty roślinnej i propozycje ochrony cennych skupień roślinności poligonu wojskowego w Biedrusku. Badania Fizjograficzne nad Polską Zachodnią, seria B, 43: 133-170.

BORYSIAK J., MELOSIK I. \& STACHNOWICZ W. 1998. Szata roślinna i ochrona torfowiska przejściowego "Gogulec" koło Poznania. Badania Fizjograficzne nad Polską Zachodnią, seria B, 47: 159-175.

BUSZKo J. 1990a. Struktura i dynamika zasięgów motyli minujących (Lepidoptera) na obszarze Doliny Dolnej Wisly. Wydawnictwo UMK, Rozprawy. 166 pp.

BuszKO J. 1990b. Studies on the mining Lepidoptera of Poland. IX. New records of Elachistidae. Polskie Pismo Entomologiczne 60: 139-151.

BUszKo J. 1993. Badania nad motylami minującymi Polski. XIV. Motyle minujące (Lepidoptera) Ojcowskiego Parku Narodowego. Wiadomości Entomologiczne 12: 201-214.

Buszko J. 2000a. Nepticulidae. In: Buszko J. \& NowACKi J.(eds), The Lepidoptera of Poland. A distributional checklist. Polish Entomological Monographs 1: 12-14.

BUszKo J. 2000b. Bucculatricidae. In: BUSzKo J. \& NOWACKI J.(eds), The Lepidoptera of Poland. A distributional checklist. Polish Entomological Monographs 1: 22.

BUSZKO J. 2000c. Gracillariidae. In: BUSZKO J. \& NOWACKI J. (eds), The Lepidoptera of Poland. A distributional checklist. Polish Entomological Monographs 1: 22-26. 
BUszKo J. 2000d. Elachistidae. In: Buszko J.\& NowACKI J. (eds), The Lepidoptera of Poland. A distributional checklist. Polish Entomological Monographs 1: 33-34.

BUSZKO J. \& BARANIAK E. 1989. Studies on the mining Lepidoptera of Poland. IV. Mining Lepidoptera of Bielinek Reserve. Polskie Pismo Entomologiczne 59: 223-234.

Buszko J., JUnNil.atnen J., KatTILA J., Nowacki J., NuPPONEN K. \& PAŁKa K. 1996. Nowe i rzadko spotykane w Polsce motyle (Lepidoptera) stwierdzone w południowo-wschodniej części kraju. Wiadomości Entomologiczne 15: $105-115$.

BUSzKO J. \& NowACKI J. (eds) 2000. The Lepidoptera of Poland. A distributional checklist. Polish Entomological Monographs, Vol. 1. Polskie Towarzystwo Entomologiczne, Poznań-Torun, 178 pp.

DORN K. 1919. Sammelbericht aus dem Warthelager bei Posen. Entomologische Jahrbuch 28: 124-133.

EISERMANN K. 2000. Ökologische Bewerkung einer ehemaligen Militärfläche: Das Tanklager Zeisigwald. Veröff. Museum für Naturkunde Chemnitz 23: 51-62.

Elsner G., HuEmER P. \& TOKAR Z. 1999. Die Palpenmotten (Lepidoptera, Gelechiidae) Mitteleuropas, Bratislava, $208 \mathrm{pp}$.

FAUNA EUROPAEA WeB SERVICE 2010. Fauna Europaea version 2.2, Available online at http://www.faunaeur.org/

HERING E. 1891. Ergänzungen und Berichtigungen zu F. O. Büttner's Pommerschen Lepidopteren. Entomologische Zeitung 52: 135-227.

IUCN 1996. Tanks and thyme. Biodiversity in former soviet military areas in Central Europe. Environmental Research Series 10: 1-136.

JAWORSKI T. 2009. Kibitnikowate (Lepidoptera: Gracillariidae) rezerwatu „Skarpa Ursynowska” w Warszawie. Wiadomości Entomologiczne 28: $53-60$.

KLAPKAREK N. \& BEUTLER H. 1999. Die Libellenfauna (Odonata) des NSG „Liberoser Endmoräne“ (Brandenburg). Märkische Entomologische Nachrichten 1: 21-38.

KONWERSKI S. 2002. Ryjkowcowate (Coleoptera: Curculionoidea: Anthribidae, Rhinomaceridae, Attelabidae, Apionidae, Curculionidae) Obszaru Chronionego Krajobrazu „Biedrusko" koło Poznania. Doctoral disseration, Zakład Zoologii Systematycznej UAM, Poznań, $156 \mathrm{pp}$.

KONWERSKI S. \& SIENKIEWICZ P. 2005. Leiodidae (Coleoptera) of the Biedrusko range in Western Poland. In: SkLODOWsKi J., HuRUK S., BARsevskis A. \& TARAsiuK S. (eds), Protection of Coleoptera in the Baltic Sea Region. Warsaw Agricultural University Press, 129-136.

KREBS CH. J. 1996. Ekologia. Eksperymentalna analiza rozmieszezenia i liczebności. Wydawnictwo Naukowe PWN, Warszawa, $735 \mathrm{pp}$.

LANDRY J.-F. \& LANDRY B. 1994. A technique for setting and mounting Microlepidoptera. Journal of the Lepidopterists' Society 48: 205-227.

MICHALSKA Z. 1988. Badania nad owadami minującymi Gór Świętokrzyskich. Wydawnictwo Naukowe UAM, Seria Zoologia Nr 13, $231 \mathrm{pp}$.

MLECZAK M. 2004. Nowe stanowiska kilku rzadkich gatunków Microlepidoptera w Polsce. Wiadomości Entomologiczne 23: 59-60.

PIEKARSKA-BONIECKA H., WILKANIEC B. 2008. Selected insect groups of shrubbery complexes of "Biedrusko" Protected Lanscape. Part II: Parasitic wasps of Pimplinae and Poemeniinae subfamily (Hymenoptera, Ichneumonidae). Polskie Pismo Entomologiczne 77: 109-124.

RAKOWSKI W. 1996. Zbiorowiska murawowe Obszaru Chronionego Krajobrazu „Biedrusko” koło Poznania. Master's dissertation Zakład Ekologii Roślin i Ochrony Środowiska UAM, Pozań, 78 pp.

RAZOWsKi J. 1973. Motyle (Lepidoptera) Polski. Część I - Ogólna. Monografie Fauny Polski, 2. Zakład Zoologii Systematycznej i Doświadczalnej, PAN, 125 pp.

Razowski J. 1990. Motyle (Lepidoptera) Polski. Część XVI. Coleophoridae. Monografie Fauny Polski 18, Instytut Systematyki i Ewolucji Zwierząt, PAN, 270 pp.

RYNARZEWSKI T. 2000. Coleophoridae. In: BUSZKO J. \& NOWACKI J. (eds), The Lepidoptera of Poland. A distributional checklist. Polish Entomological Monographs 1: 38-42.

SCHILLE F. 1931. Fauna motyli Polski. Prace monograficzne Komisji Fizjograficzne PAU, 7, 358 pp.

STACHNOWICZ W. 1996. Ląki trzęślicowe „Obszaru Chronionego Krajobrazu Biedrusko” koło Poznania. Master's disseration. Zakład Ekologii Roślin i Ochrony Środowiska UAM, Poznań, 71 pp.

STACHNOWICZ W. 1997. Osobliwości florystyczne poligonu wojskowego "Biedrusko" koło Poznania. Badania Fizjograficzne nad Polską Zachodnią, seria B, 46: 163-174.

TOLL S. 1947a. Materiały do fauny motylicznej kraju. I. Przyczynek do fauny Nepticulidów Polski. Materiały do Fizjografii Kraju 6: 1-10.

TOLL S. 1947b. Materiały do fauny motylicznej kraju. III. Przyczynek do fauny motyli tzw. drobnych Polski. Materiały do Fizjografii Kraju 6: 16-37.

TOLL S. 1950. Przyczynek do fauny motyli t. zw. drobnych (Microlepidoptera) Beskidu Ustrońskiego. Prace Biologiczne, Kraków, Nr 2, Studia nad florą i fauną Beskidu Śląskiego: 165-205.

TOLL S. 1962. Materialen zur Kenntnis der paläarktischen Arten der Familie Coleophoridae (Lepidoptera). Acta Zoologica Cracoviensia 7, 16: 577-720. 
TORo T. 1993. A cold war legacy. Wildlife Conservation 7-8: 67-71.

TROJAN P. \& WYTWER J. 1995. Różnorodność gatunkowa fauny. Mat. konf. „Nauka na rzecz różnorodności biologicznej", Warszawa 13 XII 1995. Oficyna Wydawnicza Instytut Ekologii PAN, 33-53.

VIDANO C. \& MARLETTO F. 1971. L'americana Parectopa robiniella C. (Lepidoptera Gracilariidae) nemico della Robinia in Europa. Entomologia Agraria: 12

WALCZAK U. 2002. Motyle dzienne (Lepidoptera: Papilionoidea, Hesperioidea) poligonu wojskowego w Biedrusku. Rocznik naukowy Polskiego Towarzystwa Ochrony Przyrody „Salamandra” 6: 103-118.

WILKANIEC B., BOROWIAK-SOBKOWIAK B. \& PIEKARSKA-BONIECKA H. 2008. Selected insect groups of shrubbery complexes of „Biedrusko" Protected Lanscape. Part I: Aphids (Hemiptera: Aphidoidea). Polskie Pismo Entomologiczne 77: 93-107.

WINIECKI A. 1992. Fauna poligonu „Biedrusko”. Department of Avian Biology and Ecology, Adam Mickiewicz University. Poznań [Manuscript], $45 \mathrm{pp}$.

WOCKE M. F. 1874. Verzeichniss der Falter Schlesiens. II. Microlepidoptera. Zeitschrift für Entomologie, N. F. 4: 1-107.

WOJTERsKI T., WOJTERSKA H. \& WOJTERSKA M. 1981. Potencjalna roślinność naturalna środkowej Wielkopolski. Badania Fizjograficzne nad Polską Zachodnią, Seria B, 32: 7-35.

WYRWOE E. 1994. Zbiorowiska olsowe i lęgowe kompleksów leśnych obrębu Biedrusko w nadleśnictwie Oborniki. Master's dissertation. Zakład Ekologii Roślin i Ochrony Środowiska UAM, Poznań, 88 pp.

ZIELINSKKA A. 1994. Grądy i dąbrowy kompleksów leśnych obrębu Biedrusko w nadleśnictwie Oborniki. Master's dissertation Zakład Ekologii Roślin i Ochrony Środowiska UAM, Poznań, 52 pp.

\section{STRESZCZENIE}

\section{[Motyle minujące (Lepidoptera) poligonu wojskowego "Biedrusko" (zachodnia Polska)]}

Poligon wojskowy „Biedrusko” polożony jest w bliskim sąsiedztwie Poznania (na północ od miasta) w zachodniej Polsce. Obszar ten od ponad stu lat wykorzystywany jest do celów militarnych. Teren poligonu jest w nieznacznym stopniu przeksztalcony przez czlowieka. a dominująca rolę na tym obszarze odgrywa roślinność naturalna i seminaturalna. $Z$ uwagi na duże walory przyrodnicze poligon wojskowy w Biedrusku zostal wlączony do europejskiej sieci ekologicznej Natura 2000 jako specjalny obszar ochrony siedlisk (PLH300001).

Podczas badań prowadzonych w latach 1997-2008 na terenie poligonu wojskowego "Biedrusko" stwierdzono 258 gatunków motyli minujących, co stanowi okolo $47 \%$ krajowej fauny tej grupy. Pod względem systematycznym należą one do 20 rodzin. z których najliczniejsze były Gracillariidae. Nepticulidae. Coleophoridae i Elachistidae. Po raz pierwszy wykazano z województwa wielkopolskiego 24 gatunki motyli minujących. Duże bogactwo gatunkowe motyli minujących stwierdzone na poligonie $w$ Biedrusku jest skutkiem oddziaływania kilku czynników, z których najważniejszą rolę odgrywają bogactwo florystyczne terenu i heterogenność przestrzeni. 